\title{
Prediction of pipe jacking forces using a Bayesian updating approach
}

Brian B. Sheil, Stephen K. Suryasentana, Jack O. Templeman, Bryn M. Phillips, Wen-Chieh Cheng, Limin Zhang

\section{Brian B. Sheil}

RAEng Research Fellow, Department of Engineering Science, University of Oxford, U.K. Email: brian.sheil@eng.ox.ac.uk. Corresponding author.

\section{Stephen K. Suryasentana}

Chancellor's Fellow (Lecturer), Department of Civil \& Environmental Engineering, University of Strathclyde, Glasgow G11XJ, U.K. Email: stephen.suryasentana@strath.ac.uk

\section{Jack O. Templeman}

DPhil candidate, Department of Engineering Science, University of Oxford, Oxford OX1 3PJ, U.K. Email: jack.templeman@stcatz.ox.ac.uk

Bryn M. Phillips

DPhil candidate, Department of Engineering Science, University of Oxford, Oxford OX1 3PJ, U.K. Engineer, Ward and Burke Construction Ltd.

Email: bryn.phillips@wardandburke.com

\section{Wen-Chieh Cheng}

Professor, School of Civil Engineering, Xi'an University of Architecture and Technology, Xi'an 710055, China. Shaanxi Key Laboratory of Geotechnical and Underground Space Engineering (XAUAT), Xi'an 710055, China

Email:w-c.cheng@xauat.edu.cn

\section{Limin Zhang}

Chair Professor of Geotechnical Engineering, Director of Geotechnical Centrifuge Facility, Department of Civil and Environmental Engineering, The Hong Kong University of Science and Technology, Clear Water Bay, Hong Kong.

Email: cezhangl@ust.hk

Initial submission, 28 August 2020

Revised submission, 19 January 2021

Main text word count: 4951

Tables: 5

Figures: 16
This is a peer reviewed, accepted author manuscript of the following research article: Sheil, B. B., Suryasentana, S. K., Templeman, J. O., Phillips, B. M., Cheng, W. C., \& Zhang, L. (2022). Prediction of pipejacking forces using a Bayesian updating approach. Journal of Geotechnical and Geoenvironmental Engineering, 148(1), [04021173] https://doi.org/10.1061/(ASCE)GT.1943-5606.0002645 


\section{ABSTRACT}

2 An accurate estimation of the jacking forces likely to be experienced during microtunnelling is a key

3 design concern for the design of pipe segments, the location of intermediate jacking stations and

4 the efficacy of the pipe jacking project itself. This paper presents a Bayesian updating approach for

5 the prediction of jacking forces during microtunnelling. The proposed framework is applied to two

6 pipe jacking case histories completed in the UK including a $275 \mathrm{~m}$ drive in silt and silty sand and a

71237 m drive in mudstone. To benchmark the Bayesian predictions, a 'classical' optimisation

8 technique, namely genetic algorithms, is also implemented. The results show that predictions of

9 pipe jacking forces using the prior best estimate of model input parameters provide a significant over-prediction of the monitored jacking forces for both drives. This highlights the difficulty in capturing the complex geotechnical conditions during tunnelling within prescriptive design

12 approaches and the importance of robust back-analysis techniques. Bayesian updating is also

13 shown to be a very effective option where significant improvements in the mean predictions, and

14 associated variance, of the total jacking force are obtained as more data is acquired from the drive. 


\section{INTRODUCTION}

Pipe jacking is now a mature technology and is an increasingly popular alternative to open-cut construction for the provision of below-ground utility pipelines (e.g. water, gas, sewer). There is a desire within the industry to increase the drive lengths that can be safely achieved using this technology to achieve greater economy, mainly by reducing installation of intermediate shafts e.g. Royston et al. (2016, 2020a,b). An accurate estimation of the jacking forces likely to be experienced during construction is a key design concern as it controls the design of pipe segments, the location of intermediate jacking stations and the efficacy of the pipe jacking project itself (Shou \& Jiang, 2010; Sheil et al. 2020b).

Jacking force prediction during microtunnelling has been the subject of a large body of experimental (e.g. Norris, 1992; Marshall 1998; Milligan and Norris 1999; Choo \& Ong, 2015; Pellet-Beaucour \& Kastner, 2002; Zhou et al., 2009, Sheil et al. 2016, Cheng et al. 2017; Phillips et al. 2019) and numerical (e.g. Barla \& Camusso, 2013; Cheng et al., 2007; Yen \& Shou, 2015; Ong and Choo 2016, Ji et al. 2019a) studies over the past three decades. Many investigators have sought to distill this research into closed-form prescriptive design methods. To this end, the most common approach combines the jacking force required to overcome the face pressure at the face of the tunnel boring machine (TBM) and the interface friction between the pipe string and the surrounding soil. This typically involves a combination of theoretical soil mechanics concepts and empiricism in the form of back-analysis of large case history databases, often with complementary laboratory testing. A plethora of prediction models are now available e.g. Auld (1982), Ripley (1989), Chapman and Ichioka (1999), Pellet-Beacour and Kastner (2002), Sofianos et al. (2004), Staheli (2006), Cheng et al. (2018), Zhang et al. (2018), Ji et al. (2019b). Much of this research has informed guidance in pipe jacking standards and handbooks e.g. Pipe Jacking Association (PJA, 1995), American Society of Civil Engineers (ASCE, 2001), French Society for Trenchless Technology (FSTT, 2006), Japan Microtunnelling Association (JMTA, 2000), Concrete Pipe Association of Australasia (CPAA 2013).

While significant advances have been made in prediction modelling, uncertainty surrounding the assessment of geotechnical parameters and underground conditions (e.g. karst caverns, faults, 
coal veins) remains the main barrier to accurate prediction of construction behaviour (Jin et al. 2018; Cheng et al. 2020). The observational method (Peck,1969) is an acceptable verification method for limit states to save materials, time and costs (Spross and Johansson 2017). Backanalysis of soil parameters within an observational framework has been applied widely for geotechnical applications such as slope stability (Zhang et al. 2010a, Li et al. 2016), braced excavations (Whittle et al. 1993, Hsiao et al. 2008, Juang et al. 2013) and alternative trenchless techniques such as pipe ramming (Meskele and Stuedlein 2015a). For pipe ramming applications, pipe drivability requires accurate estimates of static and dynamic soil resistance which are generally back-calculated using signal matching of stress wave measurements obtained using a pile driving analyser and interpreted using wave propagation models (e.g. Meskele and Stuedlein 2015b). In geotechnical engineering, the back-analysis process is typically cast as an optimisation problem, solved using techniques such as least squares methods (e.g. Xu and Zheng 2001), evolutionary algorithms (e.g. Chan et al. 2009, Yin et al. 2018), Bayesian approaches (e.g. Zhang et al. 2009b, 2010b, Yang et al. 2011), the maximum likelihood method (e.g. Wang et al. 2014) and more general artificial intelligence techniques such as support vector machines and artificial neural networks (Sheil et al. 2020a).

Updating uncertain geotechnical parameters during pipe jacking can be used to inform rational strategies for optimising construction operations during tunnelling. For example, uncertainty in jacking force prediction is typically alleviated using expensive inter-jacks at pre-defined intervals along the pipe string which are often not used during the drive (Atalah et al. 1994, Sheil 2020). A more flexible approach has been shown to realise major time and cost savings (Shah et al. 1993, Greene and Harkness 1995, Sterling 2020). This study presents a Bayesian approach to update uncertain model parameters for the prediction of microtunnelling jacking forces using the latest monitoring data acquired during the drive. The proposed framework is applied to two pipe jacking case histories completed in the UK including a $275 \mathrm{~m}$ drive in silt and silty sand and a $1237 \mathrm{~m}$ drive in mudstone. Predictions determined using the Bayesian updating approach are compared to those determined using 'classical' optimisation techniques for benchmarking. 
The contributions of this paper are as follows: (a) it identifies the key (uncertain) model input parameters for the prediction of jacking forces during microtunnelling, (b) it proposes a Bayesian approach to dynamically update these variables during the drive to improve predictions, (c) it compares Bayesian updating to classical optimisation techniques and identifies the relative merits of both approaches and (d) it presents the optimised variables for pipe jacking which can be used as prior estimates for future projects in similar ground conditions.

\section{DETERMINISTIC JACKING FORCE PREDICTION MODEL}

Prediction models can be broadly classified as either deterministic or probabilistic. In deterministic models, the model output is fully determined by the values of the model input parameters. This paper focuses on deterministic models such that the model predictions can be obtained as an explicit function of the model inputs. The total pipe jacking resistance comprises a face resistance and skin friction component as follows:

$$
F_{\mathrm{T}}=f_{0} \pi\left(\frac{\mathrm{D}_{\mathrm{c}}}{2}\right)^{2}+f_{S} \pi D_{p} L
$$

where $F_{\mathrm{T}}$ is the total jacking force, $f_{0}$ is the unit face resistance, $D_{\mathrm{c}}$ is the diameter of the TBM cutterhead, $f_{\mathrm{s}}$ is the unit skin friction acting along the pipe string and $D_{\mathrm{p}}$ and $L$ are the diameter and length of the pipe string respectively. An empirical model for the prediction of $f_{0}$ during microtunnelling is presented in JMTA (2000). To provide a better representation of field measurements, Shou et al. (2010) later modified that relationship as follows:

$$
f_{0}=10.0 \times 1.32 \cdot \pi \cdot D_{\mathrm{c}} \cdot N_{0}
$$

where $N_{0}$ is an empirical factor reported to vary from 1 for clayey soils to 2.5 and 3.0 for sandy and gravelly soil respectively. The widely adopted method proposed by Staheli (2006) is considered in this work for the calculation of $f_{\mathrm{s}}$ :

$$
f_{\mathrm{s}}=\frac{\tan \delta \cdot \gamma^{\prime} \cdot D_{\mathrm{p}} \cdot \cos \left(45^{\circ}+\frac{\phi^{\prime}}{2}\right)}{2 \cdot \tan \phi^{\prime}}
$$


where tan $\delta$ is the interface frictional coefficient, $\gamma^{\prime}$ is the effective unit weight of the soil and $\phi^{\prime}$ is the soil mass friction angle. For equations (1) - (3), the parameters of most interest include $N_{0}$, tanס, $\gamma^{\prime}$ and $\phi^{\prime}$ and are therefore treated as uncertain variables to be updated sequentially during the drive as shown in Fig. 1. In practice, the updating process can be implemented sequentially with a time delay until the new monitored datapoint becomes available.

\section{MARKOV CHAIN MONTE CARLO}

\section{Overview}

Deviation of model predictions from reality is typical in a wide range of geotechnical applications. A practical approach to assess the model error is through calibration with observed performances in the field or in physical model tests. Updating geotechnical model parameters within a Bayesian framework has been well-documented in the literature to reduce inconsistencies between model predictions and observed performance in laboratory testing (e.g. Kelly and Huang 2015), centrifuge modelling (e.g. Zhang et al. 2009a, 2009b, Wang et al. 2012) and field monitoring (e.g. Hsiao et al. 2008, Juang et al. 2013, Qi and Zhou 2017, Zheng et al. 2018). A common implementation of the Bayesian updating approach is to generate predictions using a deterministic model where uncertainties due to (a) soil properties, (b) geometry, (c) measurement error and (d) imperfections and approximations on the calculation model are lumped together in a model 'bias factor' applied to the deterministic prediction (e.g. Li et al. 2018). The prediction model adopted in this paper is defined as follows:

$$
\boldsymbol{y}=g(\boldsymbol{\theta})+\varepsilon
$$

where $\boldsymbol{y}=\left[y_{1}, y_{2}, \ldots, y_{n}\right]$ is the vector of observed jacking forces at jacked distances $\boldsymbol{x}=\left[x_{1}, x_{2}, \ldots, x_{n}\right]$, $n$ is the number of observed data points used for Bayesian updating and $g(\boldsymbol{\theta})$ denotes the calculated jacking force according to equations (1) - (3), and $\boldsymbol{\theta}$ is the vector of the uncertain model parameters given by:

$$
\boldsymbol{\theta}=\left[N_{0}, \tan \delta, \phi^{\prime}, \gamma^{\prime}\right]
$$


117 The parameter $\varepsilon$ is a model and measurement error term which is assumed statistically independent of the observation; this is a reasonable assumption given that measurement noise is typically caused by external factors such as electromagnetic interference from site equipment and machinery, vibrations from nearby construction works and temperature effects. It is assumed to follow a normal distribution with a mean $\mu_{\mathrm{e}}=0$ and standard deviation $\sigma_{\mathrm{e}}$ as adopted in many practical domains (Jaynes and Bretthorst 2003). Results derived from field monitoring case studies reported by O'Dwyer et al. (2019) plotted in Fig. 2 show that this is a reasonable assumption. Consequently $g(\theta)$ is no longer a deterministic value but instead a stochastic variable.

The information to be updated is a joint probability density function of multiple parameters. A key advantage of the MCMC approach is the significant flexibility in the choice of distribution for the priors. Lognormal distributions are an expedient option as they prevent unrealistic negative realisations of the non-negative geotechnical variables $\theta$ and have been widely adopted in geotechnical engineering e.g. Lumb (1966), Qi and Zhou (2017), Li et al. (2016, 2018), Zheng et al. (2018). Therefore, the uncertainty associated with the model parameters $\boldsymbol{\theta}$ are quantified as a 'prior' distribution, which is assumed to be a multivariate normal distribution with $\boldsymbol{\mu}^{\prime}{ }_{\theta}$ and $\boldsymbol{\sigma}^{\prime}{ }_{\theta}$ as the prior mean and uncertainty of the natural logarithm of $\boldsymbol{\theta}$ respectively i.e. $p\left(\boldsymbol{\theta}^{\prime}\right)=\operatorname{MVN}\left(\boldsymbol{\mu}_{\boldsymbol{\theta}}^{\prime}, \boldsymbol{\sigma}^{\prime}{ }_{\boldsymbol{\theta}}\right)$ where $\boldsymbol{\theta}^{\prime}$ is the natural logarithm of the uncertain model parameters. The prior probability distribution of the random variable $\theta^{\prime}$ is thus:

$$
p\left(\theta^{\prime} \mid \mu^{\prime}, \sigma^{\prime 2}\right)=\frac{1}{\sqrt{2 \pi \sigma^{\prime 2}}} e^{-\frac{1}{2}\left(\frac{\theta^{\prime}-\mu \prime}{\sigma^{\prime}}\right)^{2}}
$$

\section{Updating model predictions using monitored data}

The prior lognormal distributions are updated to account for the evidence provided by the monitored data to produce 'posterior' distributions of $\boldsymbol{\theta}$. The data $\boldsymbol{y}$ influences the posterior distribution through the likelihood function $p(\mathbf{y} \mid \boldsymbol{\theta}, \mathbf{x})$. This function describes the probability of predicting the observed data using the existing model for particular values of the parameter vector $\theta$ : 


$$
p(\boldsymbol{y} \mid \boldsymbol{\theta}, \boldsymbol{x})=\prod_{\mathrm{i}} p\left(y_{\mathrm{i}} \mid \boldsymbol{\theta}, \boldsymbol{x}\right)
$$
follows:

$$
p(\boldsymbol{\theta} \mid \boldsymbol{x}, \boldsymbol{y})=\frac{p(\boldsymbol{y} \mid \boldsymbol{\theta}, \boldsymbol{x}) p(\boldsymbol{\theta} \mid \boldsymbol{x})}{p(\boldsymbol{y} \mid \boldsymbol{x})}
$$
$p(\boldsymbol{y} \mid \boldsymbol{x})$ in equation (8) normalises the joint posterior distribution to ensure that it integrates to one and is obtained by marginalising out $\boldsymbol{\theta}$, as follows:

$$
p(\boldsymbol{y} \mid \boldsymbol{x})=\int p(\boldsymbol{y} \mid \boldsymbol{\theta}, \boldsymbol{x}) p(\boldsymbol{\theta} \mid \boldsymbol{x}) d \theta
$$

\section{Markov Chain Monte Carlo}

Analytical computation of equation (8) is often intractable. Instead, a robust sampling procedure known as Markov Chain Monte Carlo (MCMC) is adopted. While Metropolis-Hastings is a popular MCMC sampling procedure in geotechnical engineering (e.g. Qi and Zhou 2017, Li et al. 2017), Hamiltonian Monte Carlo (HMC) represents a more efficient alternative (Betancourt 2017). HMC sampling involves two steps: a proposal and a correction. The proposal is a stochastic perturbation of the initial parameter state, $\boldsymbol{\theta}$, while the correction rejects any proposal that strays too far away from the typical set of the target distribution. Proposals are generated using Hamiltonian dynamics by sampling from the target posterior distribution. This involves transforming the posterior density function to a Hamiltonian potential energy function and introducing a momentum variable $h_{\mathrm{j}}$ for each uncertain parameter $\theta_{i}$. This energy function is known as the canonical distribution and defines a joint distribution of $\boldsymbol{\theta}$ and $\boldsymbol{h}, p(\boldsymbol{\theta}, \boldsymbol{h} \mid \mathbf{y})$. Given the independence of these parameters, the joint distribution can be determined as the product of the posterior density, $p(\boldsymbol{\theta} \mid \mathbf{y})$, and the momentum density, $p(\boldsymbol{h})$ :

$$
p(\boldsymbol{\theta}, \boldsymbol{h} \mid \boldsymbol{y})=p(\boldsymbol{h}) p(\boldsymbol{\theta} \mid \boldsymbol{y})
$$

As there is no analytical solution for Hamilton's equations, Hamiltonian dynamics are solved using discretization integrations, typically using the 'leapfrog' method. The leapfrog approach is a time- 
163

164

165

reversible and volume-preserving numerical integrator used to propose a move to a new point in the state space. This process is desirable as it reduces the correlation between successive sampled states by proposing moves to distant states which maintain a high probability of acceptance. The HMC process can be defined as follows:

I. A candidate $\boldsymbol{h}$ is drawn from its posterior distribution, $\boldsymbol{h} \sim N(0, \boldsymbol{\Sigma})$, where $\boldsymbol{\Sigma}$ is the covariance matrix of $p(\boldsymbol{h})$.

II. The 'leapfrog' process is used to update $\boldsymbol{\theta}$ and $\boldsymbol{h}$ involving $L$ steps of size $e$ as follows:

(a) A half-step with momentum $\boldsymbol{h}$ is made using the gradient of the log-posterior density $p(\boldsymbol{\theta} \mid \boldsymbol{y})$ :

$$
h \leftarrow h+0.5 e \frac{d \log p(\boldsymbol{\theta} \mid \boldsymbol{y})}{d \boldsymbol{\theta}}
$$

(b) The parameter vector $\boldsymbol{\theta}$ is updated using $\boldsymbol{h}: \boldsymbol{\theta} \leftarrow \boldsymbol{\theta}+\mathrm{e} \boldsymbol{\Sigma}^{-1} \boldsymbol{h}$.

(c) Another half-step is made using equation (11).

III. For step $k$, the proposal is accepted with probability $\alpha$ :

$$
\alpha=\min \left(\frac{p\left(\boldsymbol{\theta}^{(k-1)} \mid \boldsymbol{y}\right) p\left(\boldsymbol{h}^{(k-1)}\right)}{p\left(\boldsymbol{\theta}^{(k)} \mid \boldsymbol{y}\right) p\left(\boldsymbol{h}^{(k)}\right)}, 1\right)
$$

If the proposal is rejected, then $\boldsymbol{\theta}^{(k)}=\boldsymbol{\theta}^{(k-1)}$.

IV. Steps I through III are repeated for $n_{\text {iter }}$ iterations in a 'chain' until convergence is achieved. In this paper, three of these chains are adopted with $n_{\text {iter }}=5000$; to enter a region of high probability, a 'burn-in' of 3000 samples is used. These parameters were found to provide an optimal balance between accuracy and computational time through trial and error.

One drawback of this technique is the need to manually tune the parameters $e$ and $L$ in the leapfrog method. This study adopts a variant of HMC, namely 'no-U-Turn sampling' (NUTS), which tunes these parameters automatically. NUTS determines the number of steps using a sophisticated tree building algorithm. The premise of NUTS is that it makes larger 'jumps' from the initial starting point into unexplored regions of the posterior distribution which achieves very efficient convergence. The NUTS implementation adapts the integration step size according to its value with respect to the acceptance ratio in equation (12) and therefore does not adopt a fixed trajectory length; it instead determines the trajectory randomly in directions opposite to the starting 
point. In the first NUTS trajectory, a single leapfrog is taken from the current state such that the trajectory has a total of two steps. The process proceeds by doubling the number of steps (to a total of four, then eight and so on) until the trajectory turns back on itself and a 'U-turn' occurs, as shown in Fig. 3. Each 'doubling' involves choosing a direction, forward or backward (in time), uniformly at random and then simulating Hamiltonian dynamics for $2^{\mathrm{j}}$ leapfrogs in that direction where $j$ is the number of leapfrogs. The total number of doublings corresponds to the 'tree depth' This framework was developed using the PyMC3 programming library in Python 3.6.

\section{CLASSICAL OPTIMISATION: GENETIC ALGORITHMS}

For the purpose of benchmarking, the same uncertain parameters $\theta$ are updated using classical optimisation procedues. Genetic algorithms (GAs) are adopted here as they represent one of the most popular optimisation techniques in geotechnical engineering e.g. Xue and Gavin (2007), Sun et al. (2008), Chan et al. (2009), Juang and Wang (2013), Yin et al. (2017). The GA approach was first proposed by Holland (1992) and was motivated by evolution and the processes of natural selection. The optimisation process of the GA is described by Fig. 4. Each iteration operates on a group of candidate solutions of the parameters to be updated, referred to collectively as a 'population'. A population size of 20 was adopted for the GA in this work. The process begins by initiating a population representing different nodes in the search space. In this paper, the initial population is generated by sampling randomly from a $99 \%$ confidence interval of the prior distributions of $\boldsymbol{\theta}$ to generate a broad search space for optimisation. In each iteration, the fitness of each individuals in the current population is evaluated using a fitness function, which in this paper is taken to be the coefficient of determination $\left(R^{2}\right)$ between the predicted and measured jacking forces up until the current jacked distance. A new population is then produced by applying genetic operations on the current solutions based on their fitness values. To improve convergence, linear scaling of the fitness values is undertaken as described in Goldberg (1989). This process is repeated until a maximum number of iterations has been achieved ( 50 was found to be optimal for this problem). 
The genetic operators achieve efficient optimization by focusing the process on solutions with high fitness; common operators are shown in Fig. 5. The initial 'selection' phase involves identifying individuals with good fitness values such that they may be preserved from population to population. The likelihood of an individual proceeding to the next population is scaled by the fitness value. The subsequent 'crossover' phase involves randomly swapping portions of the individual solutions with probability $p_{\text {cross. }}$ This operator seeks to identify an optimal variant of the individual. The final 'mutation' phase involves occasional random alteration of a chromosome with probability $p_{\text {mut. }}$ This allows the GA to retain some freedom to explore the wider search space. The GA used here was developed using Python programming, implementing each of these genetic operations in turn. The selected GA performance parameters presented in Table 1 were found to be optimal for this study and were informed by Goldberg (1989).

\section{CASE HISTORY DETAILS}

\section{Case history A: Blackpool, UK}

The first case history used to assess the proposed parameter updating framework is a $295 \mathrm{~m}$ long drive undertaken in Blackpool, UK (see Fig. 6). Figure 7 shows the ground conditions determined from two boreholes $(\mathrm{BH} 1$ and $\mathrm{BH} 2)$ located on the drive route; profiles of Standard Penetration Test $(\mathrm{N})$ value have also been superimposed on the figure. The ground conditions comprised a 0.4 $\mathrm{m}$ layer of made ground at the ground surface followed by gravelly sand $(2.6 \mathrm{~m}-4.4 \mathrm{~m})$, peat (1.0 $m-1.7 m)$, silt $(1.2 m-2.5 m)$ and silty sand/sand $(>5.0 m)$. The groundwater table was found to be $6.4 \mathrm{~m}$ above ordnance datum (OD) close to $\mathrm{BH}$ 2. The launch invert for the drive was $7.59 \mathrm{~m}$ below ground level (GL) and the overburden depth from the tunnel crown varied between $6.2 \mathrm{~m}$ and $7.8 \mathrm{~m}$. Tunnelling therefore predominantly occurred through the alternating layers of silt and silty sand. A $1.515 \mathrm{~m}$ diameter slurry-supported shield machine was used for the tunnelling whereas the diameter of the trailing concrete pipe string was $1.49 \mathrm{~m}$. The resulting $25 \mathrm{~mm}$ 'overcut' annulus was filled with a bentonite-based lubricant during tunneling to maintain tunnel bore stability and minimize friction between the soil and the pipe string. Each pipe had a weight of $35.9 \mathrm{kN}$ and a length of $2.5 \mathrm{~m}$. The buoyant uplift force acting on the pipe was calculated as $17.4 \mathrm{kN} / \mathrm{m}$. The 
output data from the TBM was recorded at $0.2 \mathrm{~m}$ intervals of jacked distance. The development of total jacking force with jacked distance monitored for the drive is presented in Fig. 8. Additional information on the project is presented in O' Dwyer et al. $(2018,2019)$.

\section{Case history B: Keswick, UK}

The second case history considered is a 1237 m drive near Keswick, Cumbria, UK (see Fig. 9(a)). The launch invert depth was $158 \mathrm{~m}$ above ordnance datum (AOD) and the overburden depth from the tunnel crown varied between approximately $5.6 \mathrm{~m}$ and $57.8 \mathrm{~m}$. Pipe-jacking was conducted in the downstream direction of the pipeline (from E-SE to W-NW) at a positive grade of $\sim 1 \%$. The tunneling was undertaken using a slurry shield machine with a $2.83 \mathrm{~m}$ diameter cutterhead and the trailing pipe string had a diameter $2.756 \mathrm{~m}$ thereby creating a $37 \mathrm{~mm}$ 'overcut'. The lubrication used was a mix of bentonite, polymer and water. Each pipe had a length of $3 \mathrm{~m}$ and a weight of $139.5 \mathrm{kN}$. The buoyant uplift force was calculated as $178.9 \mathrm{kN}$. Figure $9(\mathrm{~b})$ summarises the ground conditions as determined from six boreholes located along the length of the drive. The drive predominantly passed through extremely weak to medium strong mudstone of low abrasivity and permeability with an average unconfined compressive strength of $5.25 \mathrm{MPa}$ (coefficient of variation $=0.65)$. The groundwater table was located at $164.6 \mathrm{mAOD}$. The output data from the TBM was recorded at intervals of jacked distance varying between $0.01 \mathrm{~m}$ and $0.3 \mathrm{~m}$ for this drive; the data has therefore been resampled at $0.2 \mathrm{~m}$ for consistency with case history A. The development of total jacking force with jacked distance monitored for the drive is shown in Fig. 10. Additional details on the project are available in Phillips et al. (2019).

\section{PARAMETER UPDATING RESULTS}

\section{Case history A}

The prior best estimates of the mean and coefficient of variation (COV) of the uncertain model parameters $\boldsymbol{\theta}$ (see equation (6)) and $\varepsilon$ for case history $A$ are presented in Table 2 . Results from the parameter updating process using the MCMC approach are compared with those determined using 
the GA optimisation approach in Fig. 11; by way of example, shaded regions are used to denote the $90 \%$ confidence interval $(\mathrm{Cl})$ for the MCMC updated parameters. From Fig. 11(a), there is a gradual increase in the mean of $N_{0}$ predicted using the MCMC approach from the prior of 2.5 to approximately 5 towards the end of the drive. In contrast, predictions of $N_{0}$ using the GA approach show significant fluctuations. This is because the optimisation problem is underdetermined and there are therefore multiple combinations of $\boldsymbol{\theta}$ that can achieve an optimal solution. While the GA predicts a similar trend to MCMC initially, the two sets of predictions diverge after a jacked distance of $\sim 10 \mathrm{~m}$. This is because of the boundaries on the GA search space, defined as the $99 \% \mathrm{Cl}$ in this study. The GA has no means of 'escaping' the pre-defined search space for each parameter as new chromosomes, generated for the initial population or for mutation procedures, are drawn from the original search space. Heuristically modifying the boundaries of $\boldsymbol{\theta}$ to encompass all feasible values caused a significant increase in fluctuations leading to unrealistic parameter values. This contrasts with the MCMC approach which is free to 'ignore' the prior distribution of $\boldsymbol{\theta}$ in the presence of strong evidence which is a key advantage.

From Fig. 11(b), GA and MCMC predictions of tand are in very good agreement and indicate the prior distribution for tanð provides a significant over-prediction reflecting the effectiveness of modern pipe jacking lubrication systems. It should be noted that present predictions represent 'operational' values of tanঠ which account for lubrication-induced reductions in both the interface friction coefficient and normal effective stress exerted by the soil on the pipe. There is a slight divergence between the GA and MCMC predictions at $\sim 200 \mathrm{~m}$. This is because the GA was shown to under-predict $N_{0}$ relative to the MCMC approach in Fig. 11(a) due to the boundary on the search space. Note that MCMC predictions of the $90 \% \mathrm{Cl}$ are significant since the model does not account for the influence of stoppages on the skin friction. However, there is a gradual reduction in the $90 \%$ $\mathrm{Cl}$ as more data is acquired, particularly after $\sim 165 \mathrm{~m}$. This is because the MCMC updated model predicts $\tan \delta=0$ at this point and therefore zero skin friction. This is not unreasonable given the effectiveness of modern microtunnelling lubrication systems. For example, it is common for bentonite lubrication to form a highly effective filter cake at the soil boundary. This, in turn, allows the full hydraulic pressure of the bentonite to be exerted on the soil boundary thereby stabilising 
the tunnel bore created by the overcut of the tunnel boring machine. Even in the situation where the tunnel bore collapses on the pipe string, the significant lubrication pressures are such that appreciable effective normal stress is exerted by the soil on the pipe such that skin frictions remain low. The model therefore reduces to only the face resistance which leads to an improved certainty for $N_{0}$ due to the reduction in model complexity. In contrast, $\phi^{\prime}$ and $\gamma^{\prime}$ do not experience significant changes during MCMC updating due to the relatively strong prior, as shown in Figs. 11(c) and 11(d), respectively. While the MCMC results are quite smooth, GA predictions again fluctuate across the $99 \% \mathrm{Cl}$ search space.

MCMC predictions of the posterior variance of $\boldsymbol{\theta}$ during the drive predicted by the MCMC approach relative to the prior variance is considered further in Fig. 12. The variance of $\phi^{\prime}$ and $\gamma^{\prime}$ remain relatively unchanged during the parameter updating process due to the use of a strong prior. In contrast, the variance associated with tan $\delta$ and $N_{0}$ show significant variations during updating where both sets of results exhibit remarkably similar trends. Initial reductions in the variance are due to strong consistency of the jacking forces during the initial (un-lubricated) stages of the drive (see Fig. 8) and the variance continues to decrease gradually as more data is acquired. The sudden drop in the variance at $\sim 170 \mathrm{~m}$ is attributable to the prediction of $\tan \delta=0$ such that $F_{\mathrm{T}}$ is only a function of $N_{0}$ and the reduced uncertainty is due to the simplicity of the model. By way of example, the shape of the updated (posterior) distributions of $\boldsymbol{\theta}$ are compared to the prior distribution in Fig. 13 for selected update points during the drive. For both $N_{0}$ and tanס, the updating process (using larger and larger datasets) results in a stronger posterior where the distributions appear to achieve convergence. Figure 13(b) highlights the importance of the lognormal distribution to prevent unrealistic negative realisations of tanס. Figures $11(\mathrm{a})$ and $11(\mathrm{~b})$ clearly indicate that the operational tanס decreases significantly with the drive length. This is because the initial section of this drive was unlubricated, as shown in Fig. 8. This section becomes lubricated as tunnelling progresses, thereby lowering the average friction coefficient for the drive towards the fully lubricated case. This is evidenced by field monitoring reported elsewhere in the literature e.g. O'Dwyer et al. (2018, 2019). 
Corresponding predictions of the total jacking force for the remainder of the drive are presented in Fig. 14 using both MCMC and GA back-calculated parameters. For the MCMC analyses, in addition to the mean prediction, 500 realisations of the jacking force model obtained by sampling values of the posterior distribution of $\boldsymbol{\theta}$ are shown using light grey lines. From Fig. 14(a), prior predictions using the MCMC approach significantly over-predict the measured total jacking forces where the scatter in the predictions is also rather significant. Having acquired 50 datapoints (corresponding to a jacked distance of $10 \mathrm{~m}$ ), there is a notable reduction in the scatter of the MCMC predictions, as shown in Fig. 14(b). However, the jacking forces for the remainder of the drive are still significantly over-predicted given the initial jacking force measurements correspond to an unlubricated drive section and therefore over-predict the subsequent lubricated sections. In this instance, there is good agreement with the GA optimised prediction and the MCMC posterior mean. As more data is acquired, the MCMC posterior predictions show improved agreement with the monitored data, as shown in Figs 14(c) - 14(f) for jacked distances of $20 \mathrm{~m}-50 \mathrm{~m}$. However, there is a notable difference between the predictions determined using GA optimised parameters and the MCMC updated parameters due to the inability of the GA approach to escape the original search space.

\section{Case history $B$}

The parameter updating process for case history B is plotted in Fig. 15 as a function of jacked distance during the drive. Given the length of the drive, there is notable variability in the development of the updated value of $N_{0}$ (see Fig. 15(a)). This is partly due to the change in working pattern during the drive and the slightly variable nature of the mudstone. For jacked distances greater than approximately $450 \mathrm{~m}$, there is a divergence between the GA and MCMC predictions. This is again attributable to the inflexible GA search space (the maximum possible value for $N_{0}$ is 5.7). In contrast, there is improved agreement between GA and MCMC predictions for tand in Fig. 15(b). MCMC mean predictions of the soil friction angle, $\phi^{\prime}$, and unit weight, $\mathrm{Y}^{\prime}$, again remain constant during the updating process due to the strong prior which is in contrast with 
the GA predictions which vary within the $99 \% \mathrm{Cl}$ search space. Figure $15(\mathrm{~b})$ again shows the tendency of decreasing friction coefficient with the drive length.

The development of the posterior variance of $\boldsymbol{\theta}$ during updating, relative to the prior variance is presented in Fig. 16. While the variance of $\mathrm{y}^{\prime}$ and $\phi^{\prime}$ remain unchanged, there is a significant reduction in the variance of $N_{0}$ and tan $\delta$ as the drive progresses. The variance remains relatively high initially in the drive as the model described by equation (1) does not account for the influence of stoppages on tan $\delta$ (and consequently $N_{0}$ ).

Predictions of the development of $F_{\mathrm{T}}$ with jacked distance determined using both the MCMC and GA approaches are compared to the monitored data in Fig. 17 for salient update points during the drive. Similar to case history A, it can be seen that while the prior estimate of the parameters provides good predictions for the initial portion of the drive $(\lesssim 150 \mathrm{~m})$, a significant over-prediction is obtained for the remainder of the drive (see Fig. 17(a)). It can also be seen that there is significant scatter in the predictions, as shown by the realisations of the model, due to the prior uncertainty of the model parameters. An update of the parameters at a jacked distance of $100 \mathrm{~m}$ achieves excellent predictions for the initial portion of the drive (corresponding to the 12-hour shift working pattern), as shown in Fig. 17(b). This coincides with a reduction in the scatter of the predictions while excellent agreement between the GA and MCMC mean predictions are also obtained. However, the updated model still provides an over-prediction of the data for jacked distances $\gtrsim 400 \mathrm{~m}$ due to the change in shift working pattern. As more data is collected and used to update the model parameters, predictions for the latter stages of the drive improves significantly, as shown in Figs. 17(c) - 17(f) for updates at jacked distances $200 \mathrm{~m}-500 \mathrm{~m}$ (in increments of $100 \mathrm{~m})$.

\section{DISCUSSION}

Predictions of pipe jacking forces using the prior best estimate of model input parameters provide a significant over-prediction of the monitored jacking forces for both drives considered in this study. The trends in the back-calculated ('operational') tanס provides insight into important pipe-lubricant- 
soil mechanics during microtunnelling. For example, it is common for the initial sections of the drive to be unlubricated given the difficulty in pressurising the overcut so close to the bore exit at the launch shaft. This causes initially high frictional values which eventually tends towards a fully lubricated condition as tunnelling progresses. This reflects the effectiveness of modern lubrication systems to reduce friction during microtunnelling and suggests that the use of historical pipe jacking parameters may provide overly conservative predictions resulting in increased construction costs.

The Bayesian updating approach provides an effective means of updating model input parameters where significant improvements in the mean predictions, and associated variance, of the total jacking force were obtained as more data was acquired from the drive. While the Bayesian approach provides a smooth updating process for each model parameter, optimised parameters using the genetic algorithm approach showed significant fluctuations. Further, genetic algorithms require manual definition of the parameter search space where the $99 \%$ confidence interval of the prior distribution of parameters was adopted in this study. While Bayesian approaches have the ability to 'ignore' the prior given sufficiently strong evidence from the monitored data, the genetic algorithm was not able to venture outside the original search space. However, heuristically widening the search space to consider all feasible values can lead to unrealistic realisations of the model parameters.

The final updated/optimised parameters for both drives are compared in Table 3. The greatest discrepancy between the GA-optimised and MCMC-updated parameters relates to $N_{0}$ due to the fixed GA search space. It is noteworthy that the MCMC back-calculated mean values of $N_{0}$ (5.0 and 9.1 for sand and mudstone respectively) are significantly greater than empirical values reported by Shou et al. (2010) ( 2.5 and $>3$ respectively). This finding is not surprising given the obvious difficulty in capturing complex geotechnical conditions at the TBM face within a simplified prescriptive design approach. It does, however, highlight a need for the calibration of equation (2) using additional case histories to provide more accurate estimates of the mean and variance of $N_{0}$. MCMC and GA back-calculated values of tan $\delta$ show better agreement (ranging between 0.01 0.065). Even though existing design standards account for the reduction of interface friction 
coefficient due to the presence of lubrication, those recommendations are significantly higher than present 'operational' values (see Table 4). This is due to the combined effect of lubrication-induced reductions in the friction coefficient and effective pipe-soil contact. In contrast, the present values are much more in line with more recent empirically derived values documented elsewhere in the literature (see Table 5). These parameters can be used as more representative priors for the prediction of jacking forces in future microtunnelling drives.

\section{CONCLUSIONS}

This paper has described a Bayesian updating approach for the prediction of jacking forces during microtunnelling. The proposed framework was applied to two pipe jacking case histories completed in the UK including a $275 \mathrm{~m}$ drive in sand and silty sand and a $1237 \mathrm{~m}$ drive in mudstone. To benchmark the Bayesian predictions, a 'classical' optimisation technique, namely genetic algorithms, was also employed. Predictions of pipe jacking forces using the prior best estimate of model input parameters provided a significant over-prediction of the monitored jacking forces for both drives considered in this study. This highlights the difficulty in capturing the complex geotechnical conditions during tunnelling within prescriptive design approaches and the importance of robust back-analysis techniques. Bayesian updating was shown to be a very effective option where significant improvements in the mean predictions, and associated variance, of the total jacking force were obtained as more data was acquired from the drive. The final updated/optimised parameters for both drives were presented and have the potential to serve as the prior estimates of the model parameters for future drives of similar ground conditions.

\section{ACKNOWLEDGEMENTS}

This work was supported by the Royal Academy of Engineering under the Research Fellowship Scheme and the Engineering and Physical Sciences Research Council (grant no. EP/T006900/1)

\section{DATA AVAILABILITY STATEMENT}


Some or all data, models, or code that support the findings of this study are available from the corresponding author upon reasonable request.

\section{REFERENCES}

ASCE, 2001. 27-00 Standard Practice for Direct Design of Precast Concrete Pipe for Jacking in Trenchless Construction. Reston, Virginia.

Atalah, A. L., Bennett, D., and Iseley, T. 1994. "Estimating the required jacking force." Proc., North American No-Dig 94, NASTT, Dallas, Paper D2.

Auld, F.A. (1982). Determination of pipe jacking loads. Proceedings of the Pipe Jacking Association. Manchester.

Barla, M., \& Camusso, M. (2013). A method to design microtunnelling installations in randomly cemented Torino alluvial soil. Tunnelling and Underground Space Technology, 33, 73-81.

Betancourt, M., 2017. A conceptual introduction to Hamiltonian Monte Carlo. arXiv preprint arXiv:1701.02434.

Chan, C.M., Zhang, L.M. and Ng, J.T., 2009. Optimization of pile groups using hybrid genetic algorithms. Journal of Geotechnical and Geoenvironmental Engineering, 135(4), pp.497505.

Chapman, D.N., Ichioka, Y., 1999. Prediction of jacking forces for microtunneling operations. Trenchless Technology Research, ISTT 14 (1), 31-41

Cheng, C., Dasari, G., Chow, Y., \& Leung, C. (2007). Finite element analysis of tunnel-soil-pile interaction using displacement controlled model. Tunnelling and Underground Space Technology, 22(4), 450-466.

Cheng, W.C., Ni, J.C., Shen, J.S.L. and Huang, H.W., 2017. Investigation into factors affecting jacking force: a case study. Proceedings of the Institution of Civil Engineers-Geotechnical Engineering, 170(4), pp.322-334.

Cheng, W.C., Ni, J.C., Arulrajah, A. and Huang, H.W., 2018. A simple approach for characterising tunnel bore conditions based upon pipe-jacking data. Tunnelling and Underground Space Technology, 71, pp.494-504.

Cheng, W.C., Bai, X.D., Sheil, B.B., Li, G. and Wang, F., 2020. Identifying characteristics of pipejacking parameters to assess geological conditions using optimisation algorithm-based support vector machines. Tunnelling and Underground Space Technology, 106, p.103592.

Choo, C., \& Ong, D. (2015). Evaluation of pipe-jacking forces based on direct shear testing of reconstituted tunneling rock spoils. Journal of Geotechnical and Geoenvironmental Engineering, 141(10), 04015044.

Concrete Pipe Association of Australasia (2013) “Design Manual-Jacking Design Guidelines”.

French Society for Trenchless Technology (FSTT), 2006. Microtunneling and Horizontal Drilling: Recommendations. ISTE, Newport Beach.

Goldberg, D.E., 1989. Genetic algorithms in search. Optimization, and MachineLearning.

Greene, B.H., and Harkness, A. 1995. Installation of a large diameter reinforced concrete pipe by jacking methods. Environmental \& Engineering Geoscience, 1: 518-523. doi:10.2113/gseegeosci.l.4.518.

Harr, M. E. (1984). "Reliability-based design in civil engineering." 1984 Henry M. Shaw Lecture, Dept. of Civil Engineering, North Carolina State University, Raleigh, N.C.

Holland, J.H., 1992. Genetic algorithms. Scientific american, 267(1), pp.66-73. 
Hsiao, E. C. L., Schuster, M., Juang, C. H., and Kung, T. C. (2008). "Reliability analysis of excavation-induced ground settlement for building serviceability evaluation." J. Geotech. Geoenviron. Eng., 134(10), 1448-1458.

Japan Micro Tunneling Association, 2013. Pipe-Jacking Application. JMTA, Tokyo.

Jaynes, E. T. and G. L. Bretthorst. 2003. Probability theory: The logic of science. Cambridge, UK: Cambridge University Press

$\mathrm{Ji}, \mathrm{X}$., Ni, P. and Barla, M., 2019a. Analysis of jacking forces during pipe jacking in granular materials using particle methods. Underground Space, 4(4), pp.277-288.

Ji, X., Zhao, W., Ni, P., Barla, M., Han, J., Jia, P., Chen, Y. and Zhang, C., 2019b. A method to estimate the jacking force for pipe jacking in sandy soils. Tunnelling and Underground Space Technology, 90, pp.119-130.

Jin, Y., Biscontin, G. and Gardoni, P., 2018. A Bayesian definition of 'most probable' parameters. Geotechnical Research, 5(3), pp.130-142.

Juang $\mathrm{CH}$, Luo Z, Atamturktur S, Huang HW. Bayesian updating of soil parameters for braced excavations using field observations. J Geotech Geoenviron Eng 2013;139(3):395-406.

Juang, C.H. and Wang, L., 2013. Reliability-based robust geotechnical design of spread foundations using multi-objective genetic algorithm. Computers and Geotechnics, 48, pp.96-106.

Kelly, R. and Huang, J., 2015. Bayesian updating for one-dimensional consolidation measurements. Canadian Geotechnical Journal, 52(9), pp.1318-1330.

Kulhawy, F. H. (1992). "On the evaluation of soil properties.” ASCE Geotech. Spec. Publ. No. 31, 95-115.

Li, X.Y., Zhang, L.M., Jiang, S.H., Li, D.Q. and Zhou, C.B., 2016. Assessment of slope stability in the monitoring parameter space. Journal of Geotechnical and Geoenvironmental Engineering, 142(7), p.04016029.

Li, J., Hu, P., Uzielli, M. and Cassidy, M.J., 2018. Bayesian prediction of peak resistance of a spudcan penetrating sand-over-clay. Géotechnique, 68(10), pp.905-917.

Lumb, P., 1966. The variability of natural soils. Canadian Geotechnical Journal, 3(2), pp.74-97.

Marshall, M.A., 1998. Pipe-jacked tunnelling: jacking loads and ground movements (Doctoral dissertation). University of Oxford.

Meskele, T. and Stuedlein, A.W., 2015a. Static soil resistance to pipe ramming in granular soils. Journal of Geotechnical and Geoenvironmental Engineering, 141(3), p.04014108.

Meskele, T. and Stuedlein, A.W., 2015b. Drivability analyses for pipe-ramming installations. Journal of Geotechnical and Geoenvironmental Engineering, 141(3), p.04014107.

Milligan, G.W.E., Norris, P., 1999. Pipe-soil interaction during pipe jacking. Proc. Inst. Civil Eng.Geotech. Eng. 137 (1), 27-44.

Namli, M. and Guler, E., 2017. Effect of bentonite slurry pressure on interface friction of pipe jacking. Journal of Pipeline Systems Engineering and Practice, 8(2), p.04016016.

Norris, P., 1992. The behaviour of jacked concrete pipes during site installation (Doctoral dissertation). University of Oxford.

O'Dwyer, K.G., McCabe, B.A., Sheil, B.B. \& Hernon, D.P. (2018) Blackpool South Strategy Project: analysis of pipe jacking records. Submitted for Civil Engineering Research in Ireland (CERI), Dublin, Ireland.

O'Dwyer, K.G., McCabe, B.A. and Sheil, B.B., 2019. Interpretation of pipe-jacking and lubrication records for drives in silty soil. Underground Space. 
Ong, D.E.L. and Choo, C.S., 2016. Back-analysis and finite element modeling of jacking forces in weathered rocks. Tunnelling and Underground Space Technology, 51, pp.1-10.

Pal, M. and Deswal, S., 2008. Modeling pile capacity using support vector machines and generalized regression neural network. Journal of geotechnical and geoenvironmental engineering, 134(7), pp.1021-1024.

Peck, R.B., 1969. Advantages and limitations of the observational method in applied soil mechanics. Geotechnique, 19(2), pp.171-187.

Pellet-Beaucour, A.-L., \& Kastner, R. (2002). Experimental and analytical study of friction forces during microtunneling operations. Tunnelling and Underground Space Technology, 17(1), 83-97.

Phillips, B.M., Royston, R., Sheil, B.B. and Byrne, B.W., 2019. Instrumentation and Monitoring of a Concrete Jacking Pipe. In International Conference on Smart Infrastructure and Construction 2019 (ICSIC) Driving data-informed decision-making (pp. 457-462). ICE Publishing.

Pipe Jacking Association, 1995. Guide to Best Practice for the Installation of Pipe Jacks and Microtunnels. Pipe Jacking Association, London.

Qi, X.H. and Zhou, W.H., 2017. An efficient probabilistic back-analysis method for braced excavations using wall deflection data at multiple points. Computers and Geotechnics, 85, pp.186-198.

Reilly, C. C. \& Orr, T. L. L. (2012). Analysis of interface friction effects on microtunnel jacking forces in coarse-grained soils. Proceedings of the Bridge and Concrete Research in Ireland Conference. Dublin. pp. 121-126.

Ripley, K.J., 1989. The performance of jacked pipes. PhD thesis. University of Oxford.

Royston R, Phillips BM, Sheil BB and Byrne BW (2016) Bearing capacity beneath tapered blades of open dug caissons in sand. Proceedings of Civil Engineering Research in Ireland 2016 Conference, Galway, Ireland (Jamie G (ed.)). Civil Engineering Research Association of Ireland, pp. 473-478.

Royston, R., B. Sheil, B. and W. Byrne, B., 2020a. Monitoring the construction of a large-diameter caisson in sand. Proceedings of the Institution of Civil Engineers-Geotechnical Engineering, pp.1-17.

Royston, R., Sheil, B.B. and Byrne, B.W., 2020b. Undrained bearing capacity of the cutting face of large-diameter caissons. Géotechnique.

Shah, D.D., Jain, S.K. and Prybella Jr, R.W., 1993. Performance of remotely controlled fiberglass pipe jacking system. Journal of construction engineering and management, 119(4), pp.832851.

Sheil, B.B., Curran, B. \& McCabe, B.A. (2016) Recent experiences of utility microtunnelling in Irish limestone, mudstone and sandstone rock. Tunnelling \& Underground Space Technology. 51: 326-337.

Sheil, B., 2020. Prediction of microtunnelling jacking forces using a probabilistic observational approach. Tunnelling and Underground Space Technology, 109, p.103749.

Sheil, B.B., Suryasentana, S.K., Mooney, M.A. and Zhu, H., 2020a. Machine learning to inform tunnelling operations: recent advances and future trends. Proceedings of the Institution of Civil Engineers-Smart Infrastructure and Construction, pp.1-22.

Sheil, B.B., Suryasentana, S.K. and Cheng, W.C., 2020b. Assessment of Anomaly Detection Methods Applied to Microtunneling. Journal of Geotechnical and Geoenvironmental Engineering, 146(9), p.04020094.

Shou, K., Yen, J. and Liu, M., 2010. On the frictional property of lubricants and its impact on jacking force and soil-pipe interaction of pipe-jacking. Tunnelling and Underground Space Technology, 25(4):469-477. 
Shou, K.J. and Jiang, J.M., 2010. A study of jacking force for a curved pipejacking. Journal of Rock Mechanics and Geotechnical Engineering, 2(4), pp.298-304.

Sofianos, A.I., Loukas, P., Chantzakos, Ch., 2004. Pipe jacking a sewer under Athens.Tunneling and Underground Space Technology 19 (2), 83-97.

Spross, J. and Johansson, F., 2017. When is the observational method in geotechnical engineering favourable?. Structural safety, 66, pp.17-26.

Staheli, K., 2006. Jacking Force Prediction: An Interface Friction Approach Based OnPipe Surface Roughness, A Dissertation in Civil and Environmental Engineering,Georgia Institute of Technology.

Stein, D., Möllers, K. and Bielecki, R., 1989. Microtunnelling. Vch Verlagsgesellschaft Mbh.

Sterling, R.L., 2020. Developments and research directions in pipe jacking and microtunneling. Underground Space, 5(1), pp.1-19.

Sun, J., Li, J. and Liu, Q., 2008. Search for critical slip surface in slope stability analysis by splinebased GA method. Journal of geotechnical and geoenvironmental engineering, 134(2), pp.252-256.

Wang, L., Ravichandran, N. and Juang, C.H., 2012. Bayesian updating of KJHH model for prediction of maximum ground settlement in braced excavations using centrifuge data. Computers and Geotechnics, 44, pp.1-8.

Wang, L., Luo, Z., Xiao, J. H., and Juang, C. H. (2014). "Probabilistic inverse analysis of excavation-induced wall and ground responses for assessing damage potential of adjacent buildings." Geotech. Geol. Eng., 32(2), 273-285.

Whittle, A. J., Hashash, Y. M. A., and Whitman, R. V. (1993). "Analysis of deep excavations in Boston." J. Geotech. Eng., 119(1), 69-90.

$\mathrm{Xu}$, J., and Zheng, Y. R. (2001). "Random back analysis of field geotechnical parameter by response surface method." Rock Soil Mechanics, 22(2), 167-170.

Xue, J.F. and Gavin, K., 2007. Simultaneous determination of critical slip surface and reliability index for slopes. Journal of Geotechnical and Geoenvironmental Engineering, 133(7), pp.878-886.

Yang, C. X., Wu, Y. H., Hon, T., and Feng, X. T. (2011). "Application of extended Kalman filter to back analysis of the natural stress state accounting for measuring uncertainties." Int. J. Numer. Anal. Meth. Geomech., 35(6), 694-712.

Ye, Y., Peng, L., Zhou, Y., Yang, W., Shi, C. and Lin, Y., 2020. Prediction of Friction Resistance for Slurry Pipe Jacking. Applied Sciences, 10(1), p.207.

Yen, J., \& Shou, K. (2015). Numerical simulation for the estimation the jacking force of pipe jacking. Tunnelling and Underground Space Technology, 49, 218-229.

Yin, Z.Y., Jin, Y.F., Shen, J.S. and Hicher, P.Y., 2018. Optimization techniques for identifying soil parameters in geotechnical engineering: comparative study and enhancement. International Journal for Numerical and Analytical Methods in Geomechanics, 42(1), pp.70-94.

Zhang, P., Behbahani, S. S., Ma, B., Iseley, T. \& Tan, L. (2018). A jacking force study of curved steel pipe roof in Gongbei tunnel: Calculation review and monitoring data analysis. Tunnelling and Underground Space Technology, 72, 305-322.

Zhang, L.L., Tang, W.H. and Zhang, L.M., 2009a. Bayesian model calibration using geotechnical centrifuge tests. Journal of geotechnical and geoenvironmental engineering, 135(2), pp.291-299.

Zhang, J., Zhang, L. M., and Tang, W. H. (2009b). "Bayesian framework for characterizing geotechnical model uncertainty.” J. Geotech. Geoenviron. Eng.J. Geotech. Geoenviron. Eng., 10.1061/(ASCE)GT.1943- 5606.0000018, 932-940. 
619

620

621

622

623

624

625

626

627

628

629

630

631

632

Zhang, J., Tang, W. H., and Zhang, L. M. (2010a). "Efficient probabilistic back-analysis of slope stability model parameters." J. Geotech. Geoenviron. Eng., 10.1061/(ASCE)GT.19435606.0000205, 99-109.

Zhang LL, Zhang J, Zhang LM, Tang WH. Back analysis of slope failure with Markov chain Monte Carlo simulation. Comput Geotech 2010b;37(7-8):905-12.

Zhang, P., Behbahani, S.S., Ma, B., Iseley, T. and Tan, L., 2018. A jacking force study of curved steel pipe roof in Gongbei tunnel: Calculation review and monitoring data analysis. Tunnelling and Underground Space Technology, 72, pp.305-322.

Zheng, D., Huang, J., Li, D.Q., Kelly, R. and Sloan, S.W., 2018. Embankment prediction using testing data and monitored behaviour: A Bayesian updating approach. Computers and Geotechnics, 93, pp.150-162.

Zhou, S., Wang, Y., \& Huang, X. (2009). Experimental study on the effect of injecting slurry inside a jacking pipe tunnel in silt stratum. Tunnelling and Underground Space Technology, 24(4), $466-471$. 
Table 1 Genetic algorithm performance parameters adopted in this study based on Goldberg (1989).

\begin{tabular}{ll}
\hline Parameter & Value \\
\hline Number of individuals per population, $n_{\text {sol }}$ & 20 \\
Number of generations, $n_{\text {gen }}$ & 50 \\
Probability of crossover, $p_{\text {cross }}$ & 0.6 \\
Probability of mutation, $p_{\text {mut }}$ & 0.05 \\
\hline
\end{tabular}

Table 2 Prior distributions of model parameters

\begin{tabular}{|c|c|c|c|c|c|}
\hline \multirow[t]{2}{*}{ Parameter } & \multicolumn{2}{|c|}{ Mean, $\boldsymbol{\mu}$} & \multirow{2}{*}{$\begin{array}{c}\text { Coefficient } \\
\text { of variation, } \\
\text { COV }\end{array}$} & \multirow[b]{2}{*}{ Distribution } & \multirow[b]{2}{*}{ Basis } \\
\hline & $\begin{array}{c}\text { Case } \\
\text { history A }\end{array}$ & $\begin{array}{c}\text { Case } \\
\text { history B }\end{array}$ & & & \\
\hline $\begin{array}{l}\text { Empirical face } \\
\text { resistance } \\
\text { factor, } N_{0}\end{array}$ & 2.5 & 3.5 & 0.2 & Lognormal & $\begin{array}{l}\text { Mean based on Shou et al. } \\
\text { (2010); weak COV assumed }\end{array}$ \\
\hline \multirow{2}{*}{$\begin{array}{l}\text { Interface friction } \\
\text { coefficient, tan } \delta\end{array}$} & \multirow{2}{*}{0.15} & \multirow{2}{*}{0.15} & \multirow{2}{*}{0.86} & \multirow{2}{*}{ Lognormal } & $\begin{array}{l}\text { Mean and COV based on } \\
\text { measurements reported by }\end{array}$ \\
\hline & & & & & $\begin{array}{l}\text { Staheli (2006), Shou et al. } \\
\text { (2010), Namli \& Guler (2017) }\end{array}$ \\
\hline $\begin{array}{l}\text { Soil friction } \\
\text { angle, } \phi^{\prime}\left({ }^{\circ}\right)\end{array}$ & 32 & 45 & 0.075 & Lognormal & $\begin{array}{l}\text { Mean based on site } \\
\text { measurements; COV based on } \\
\text { Harr (1984), Kulhawy (1992) }\end{array}$ \\
\hline $\begin{array}{l}\text { Soil unit weight, } \\
\mathrm{V}^{\prime}\left(\mathrm{kN} / \mathrm{m}^{3}\right)\end{array}$ & 18 & 25 & 0.05 & Lognormal & $\begin{array}{l}\text { Mean based on site } \\
\text { measurements; COV based on } \\
\text { Harr (1984), Kulhawy (1992) }\end{array}$ \\
\hline $\begin{array}{l}\text { Model and } \\
\text { measurement } \\
\text { error, } \varepsilon(\mathrm{kN})\end{array}$ & 100 & 100 & 0.2 & Normal & $\begin{array}{l}\text { Mean based on monitored data } \\
\text { reported by O'Dwyer et al. } \\
\text { (2019); weak COV assumed }\end{array}$ \\
\hline
\end{tabular}


Table 3 Optimised model parameters for case histories A and B at final update

\begin{tabular}{|c|c|c|c|c|c|c|c|c|}
\hline \multirow[t]{2}{*}{ Parameter } & \multicolumn{4}{|c|}{ Case history A } & \multicolumn{4}{|c|}{ Case history B } \\
\hline & Prior & GA & $\begin{array}{l}\text { MCMC } \\
\text { mean }\end{array}$ & $\begin{array}{c}\text { MCMC } \\
\text { COV }\end{array}$ & Prior & GA & $\begin{array}{l}\text { MCMC } \\
\text { mean }\end{array}$ & $\begin{array}{c}\text { MCMC } \\
\text { COV }\end{array}$ \\
\hline $\begin{array}{l}\text { Empirical face } \\
\text { resistance } \\
\text { factor, } N_{0}\end{array}$ & 2.5 & 2.86 & 5.04 & 0.04 & 3.5 & 5.19 & 9.1 & 0.03 \\
\hline $\begin{array}{l}\text { Interface } \\
\text { friction } \\
\text { coefficient, } \\
\text { tanס }\end{array}$ & 0.15 & 0.065 & 0.01 & 0.39 & 0.15 & 0.055 & 0.048 & 0.20 \\
\hline $\begin{array}{l}\text { Soil friction } \\
\text { angle, } \phi^{\prime}\left({ }^{\circ}\right)\end{array}$ & 32 & 37.0 & 33.6 & 0.08 & 45 & 45.6 & 45.9 & 0.07 \\
\hline $\begin{array}{l}\text { Soil unit } \\
\text { weight, } \mathrm{y}^{\prime} \\
\left(\mathrm{kN} / \mathrm{m}^{3}\right)\end{array}$ & 18 & 17.9 & 17.8 & 0.05 & 25 & 24.3 & 25.1 & 0.05 \\
\hline $\begin{array}{l}\text { Model and } \\
\text { measurement } \\
\text { error standard } \\
\text { deviation, } \varepsilon \\
(\mathrm{kN})\end{array}$ & 100 & - & 78.9 & 0.02 & 100 & - & 758.4 & 0.01 \\
\hline
\end{tabular}

Table 4 Pipe jacking friction coefficient recommended by various design standards

\begin{tabular}{llll}
\hline Standard & $\tan \delta$ & Case history A & Case history B \\
\hline JMTA (Japan) & $\tan \left(\phi^{\prime} / 2\right)$ & 0.29 & 0.41 \\
GB 50332 (China) & $\tan \left(30^{\circ}\right)$ & 0.58 & 0.58 \\
ATV A 161 (Germany) & $\tan \left(\phi^{\prime} / 2\right)$ & 0.29 & 0.41 \\
ASTN F 1962 (North America) & $\tan \left(\phi^{\prime} / 2\right)$ & 0.29 & 0.41 \\
BS EN 1594 (UK) & $\tan \left(\phi^{\prime}\right)$ & 0.62 & 1 \\
PJA (1995; UK) & $\tan \left(\phi^{\prime}\right)$ & 0.62 & 1 \\
\hline
\end{tabular}


Table 5 Selected pipe jacking friction coefficients reported in in the literature

\begin{tabular}{|c|c|c|c|}
\hline Reference & Ground conditions & Lubrication & $\begin{array}{l}\text { Friction } \\
\text { coefficient, tanס }\end{array}$ \\
\hline Stein et al. (1989) & Various & Lubrication used & $0.1-0.3$ \\
\hline Pellet-Beaucour \& Kastner (2002) & Sandy clays & Bentonite & $0.07-0.09$ \\
\hline Pellet-Beaucour \& Kastner (2002) & Sands and gravels & Bentonite & $0.17-0.5$ \\
\hline Pellet-Beaucour \& Kastner (2002) & Sands & Bentonite & $0.03-0.14$ \\
\hline Staheli (2006) & Very dense well-graded sand & Bentonite 'mass application' & 0.06 \\
\hline Staheli (2006) & Silty sand & Bentonite 'mass application' & 0.05 \\
\hline Staheli (2006) & $\begin{array}{l}\text { Poorly graded sands with gravel and well-graded } \\
\text { sands and gravels }\end{array}$ & Bentonite 'mass application' & 0.06 \\
\hline Staheli (2006) & $\begin{array}{l}\text { Poorly graded sands with gravel and well-graded } \\
\text { sands and gravels }\end{array}$ & Bentonite 'controlled application' & 0.5 \\
\hline Staheli (2006) & $\begin{array}{l}\text { Poorly graded sands with gravel and well-graded } \\
\text { sands and gravels }\end{array}$ & Bentonite 'mass application' & 0.05 \\
\hline Staheli (2006) & Silty sand & Bentonite 'controlled application' & 0.2 \\
\hline Staheli (2006) & Silty sand & Bentonite 'mass application' & 0.04 \\
\hline Shou et al. (2010) & Gravel formation & Bentonite & 0.09 \\
\hline Shou et al. (2010) & Gravel formation & Bentonite and polymer & 0.06 \\
\hline Reilly \& Orr (2012) & Slightly silty sand and sandy gravel & Bentonite & $0.08-0.12$ \\
\hline Reilly \& Orr (2012) & Sand & Bentonite & $0.1-0.14$ \\
\hline Choo \& Ong (2015) & Sandstone & Bentonite & 0.31 \\
\hline Choo \& Ong (2015) & Shale & Bentonite & 0.2 \\
\hline Choo \& Ong (2015) & Phyllite & Bentonite & 0.07 \\
\hline Choo \& Ong (2015) & Shale & Bentonite & 0.71 \\
\hline Cheng et al. (2017) & $90 \%$ fine soil governed sand or gravel deposit & Bentonite with $2 \%$ polymer & 0.07 \\
\hline Cheng et al. (2017) & $\begin{array}{l}50 \% \text { gravel formation and } 50 \% \text { fine soil governed } \\
\text { sand deposit }\end{array}$ & Bentonite with $2 \%$ polymer & 0.03 \\
\hline Cheng et al. (2017) & Gravel formation & Bentonite with $2 \%$ polymer & $0.02-0.8$ \\
\hline Cheng et al. (2017) & $\begin{array}{l}20 \% \text { gravel formation and } 80 \% \text { fine soil governed } \\
\text { sand or gravel deposit }\end{array}$ & Bentonite with $2 \%$ polymer & 0.06 \\
\hline Cheng et al. (2018) & Fine soil governed gravel or sand deposit & Bentonite with $2 \%$ polymer & $0.52-0.59$ \\
\hline Zhang et al. (2018) & Gravelly coarse sand & Lubrication used, details not provided & 0.1 \\
\hline Ye et al. (2020) & Clays - sands & Lubrication used, details not provided & 0.01 \\
\hline
\end{tabular}






Fig. 1 Parameter updating process for jacking force prediction models 


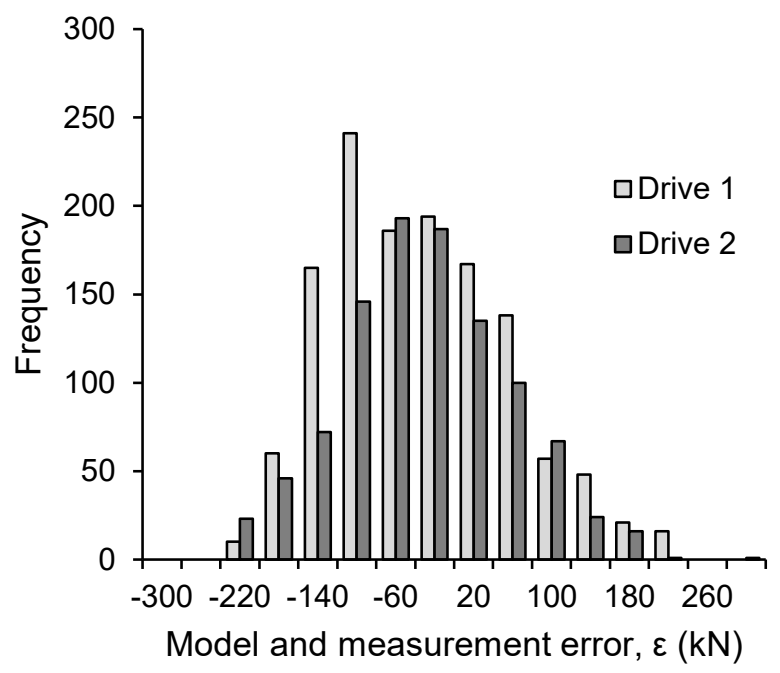

Fig. 2 Model and measurement error derived from case histories reported in O'Dwyer et al. (2019)
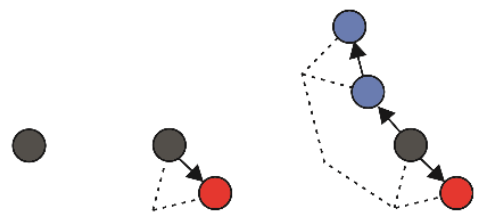

Start Leapfrog $1 \quad$ Leapfrog 2

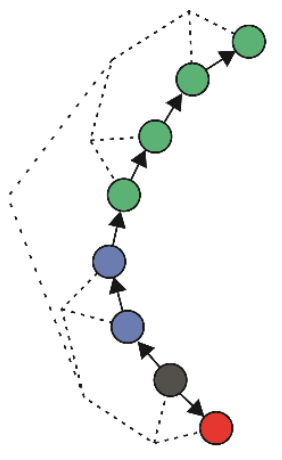

Leapfrog 3

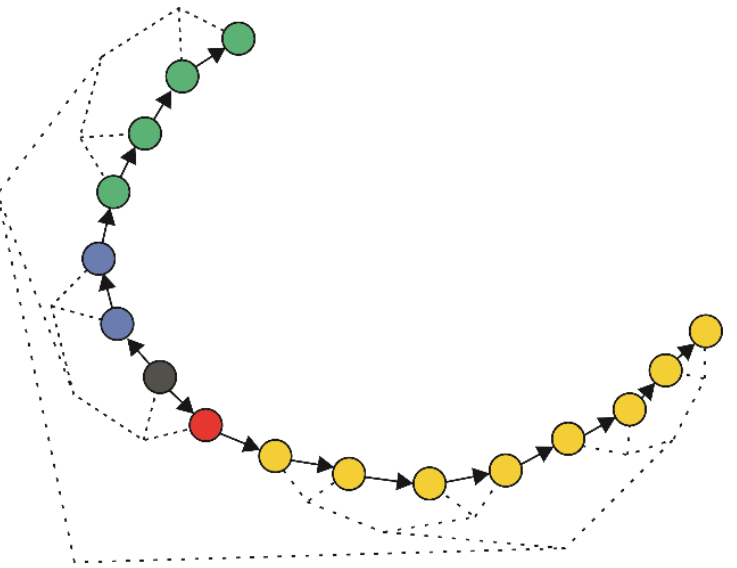

Leapfrog 4

Fig. 3 Illustration of two-dimensional 'no U-turn' trajectory via repeated doubling with corresponding binary tree shown using dashed lines. In this example, the chosen directions were forward (red node), backward (blue nodes), backward (green nodes), forward (yellow nodes) 


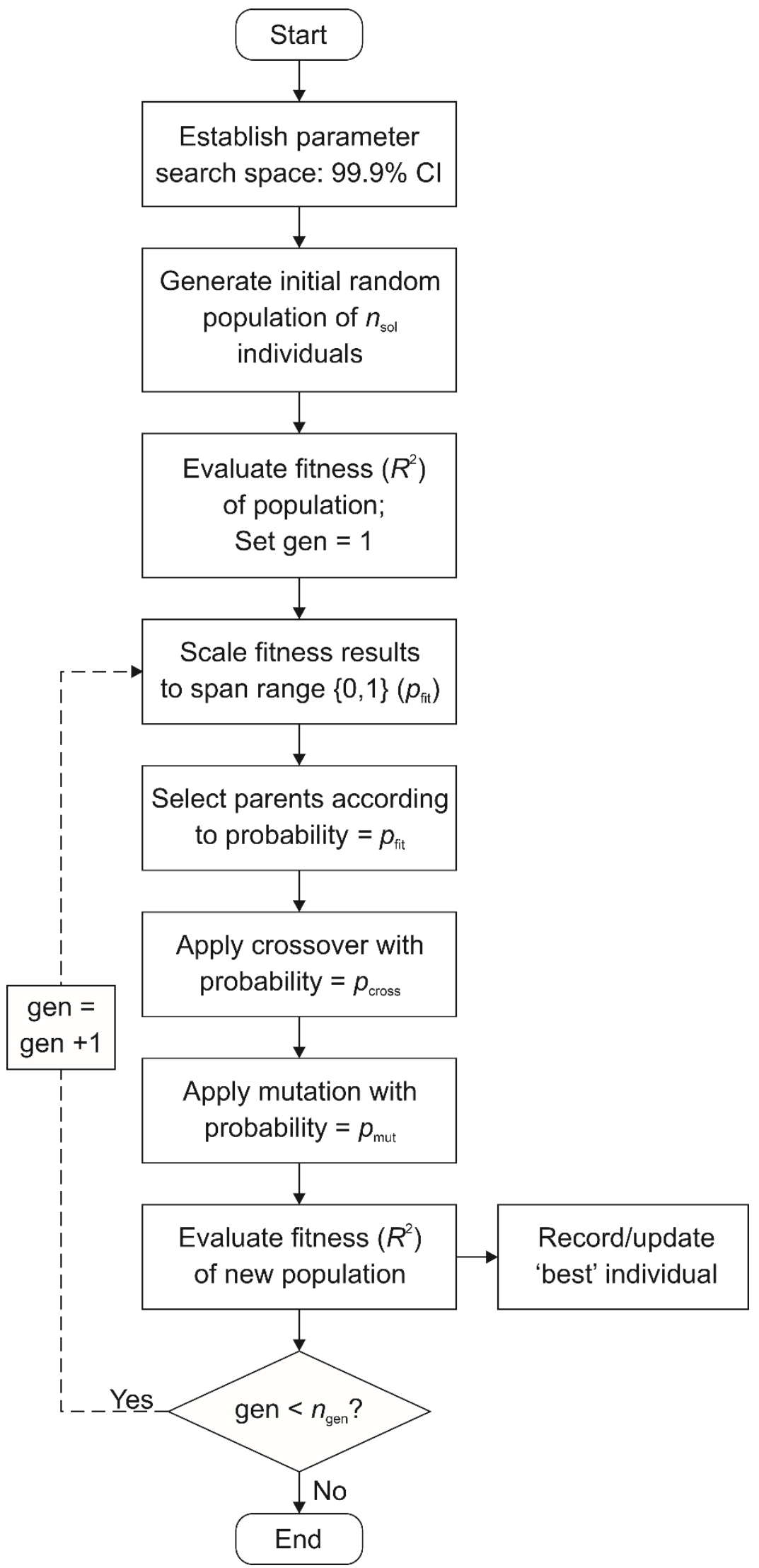

Fig. 4 Workflow for genetic algorithm developed in this work 


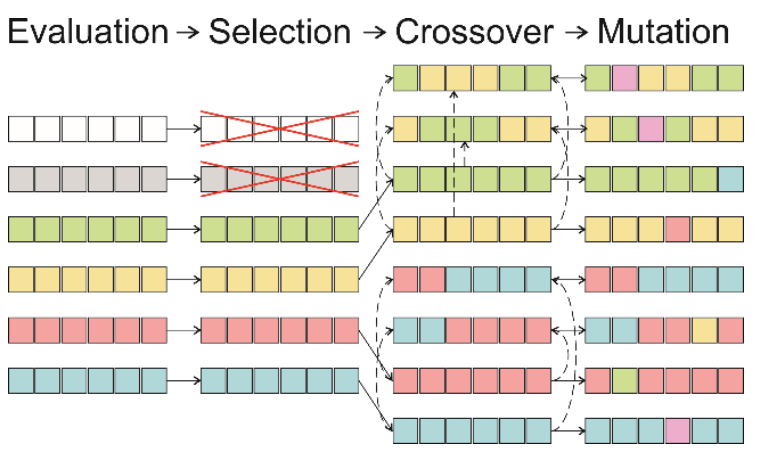

Fig. 5 Overview of genetic operators used for genetic algorithm optimisation procedure

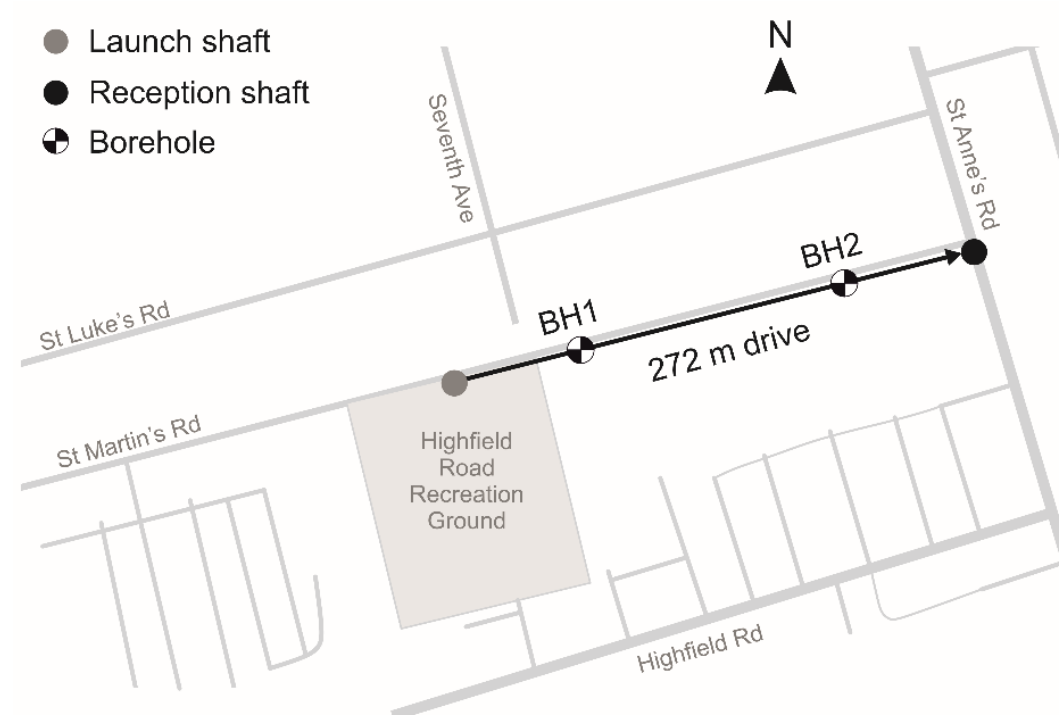

Fig. 6 Location of tunnels and shafts at the case history A site 


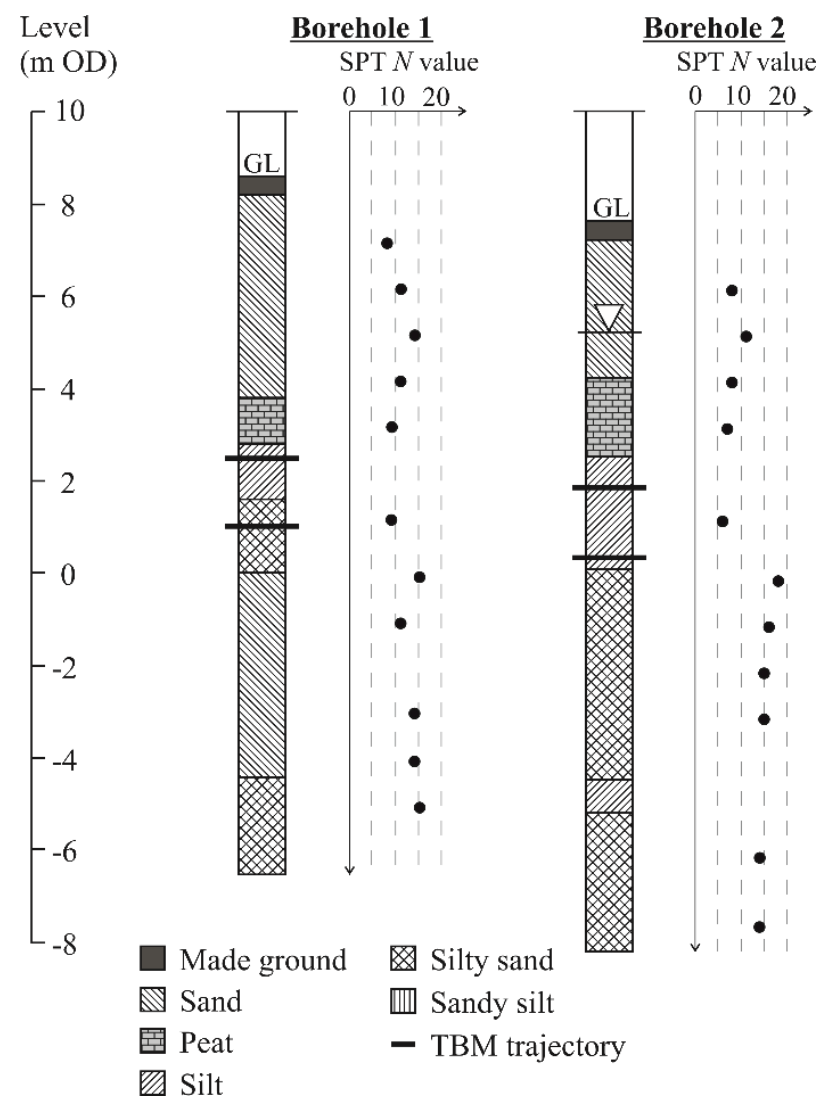

Fig. 7 Stratigraphy at the boreholes along the tunnel drive as well as the associated uncorrected SPT profiles for case history A

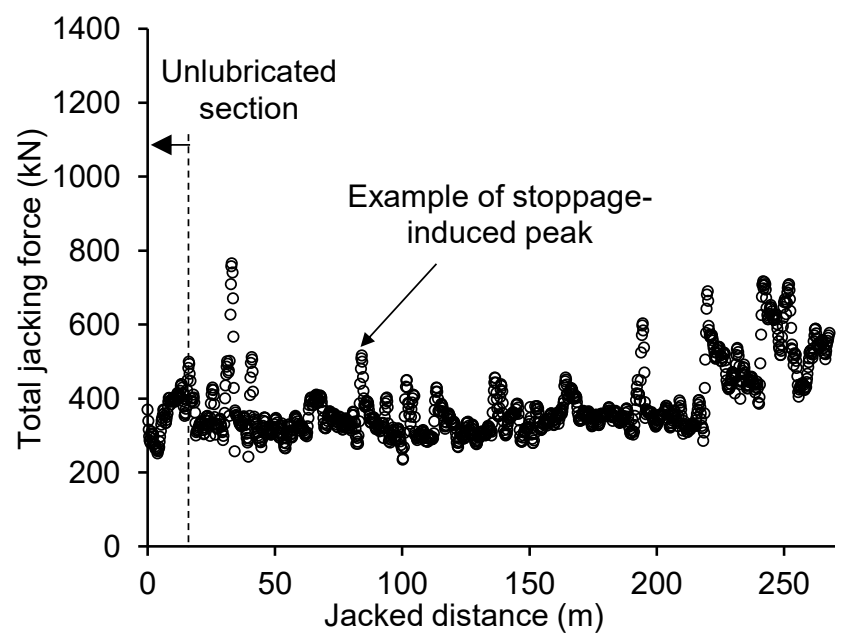

Fig. 8 Monitored data for case history A 
(a)

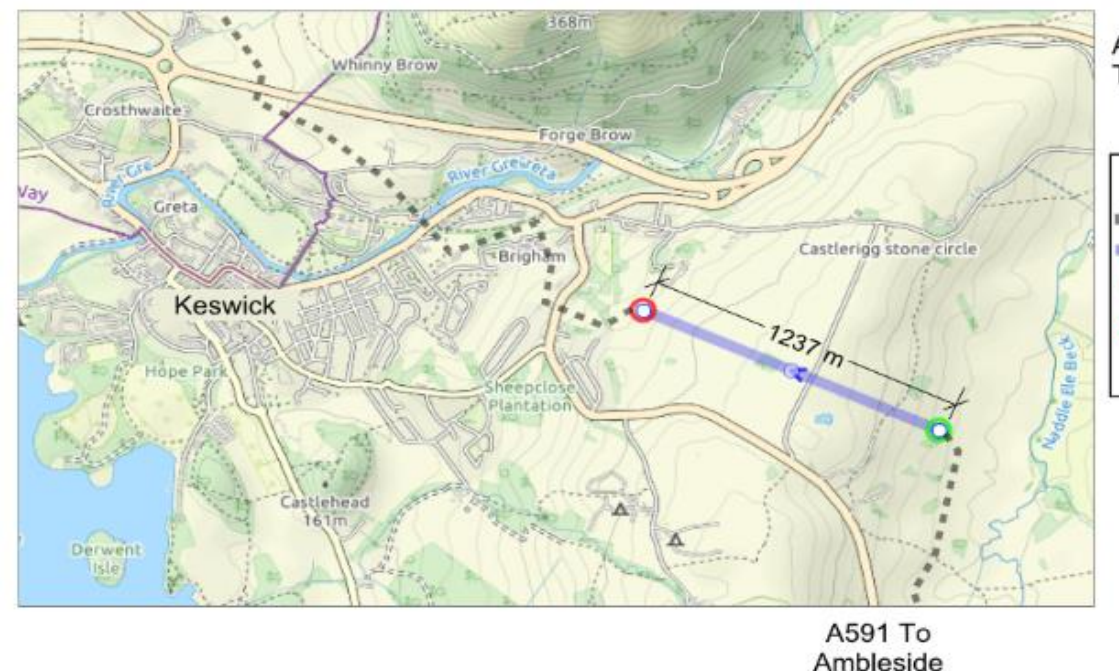

A66

To Penrith

\begin{tabular}{|l|} 
Key \\
= = Pipeline route \\
$=$ = Tunnel drive \\
= Launch shaft \\
location \\
o Reception shaft \\
location
\end{tabular}

(b)

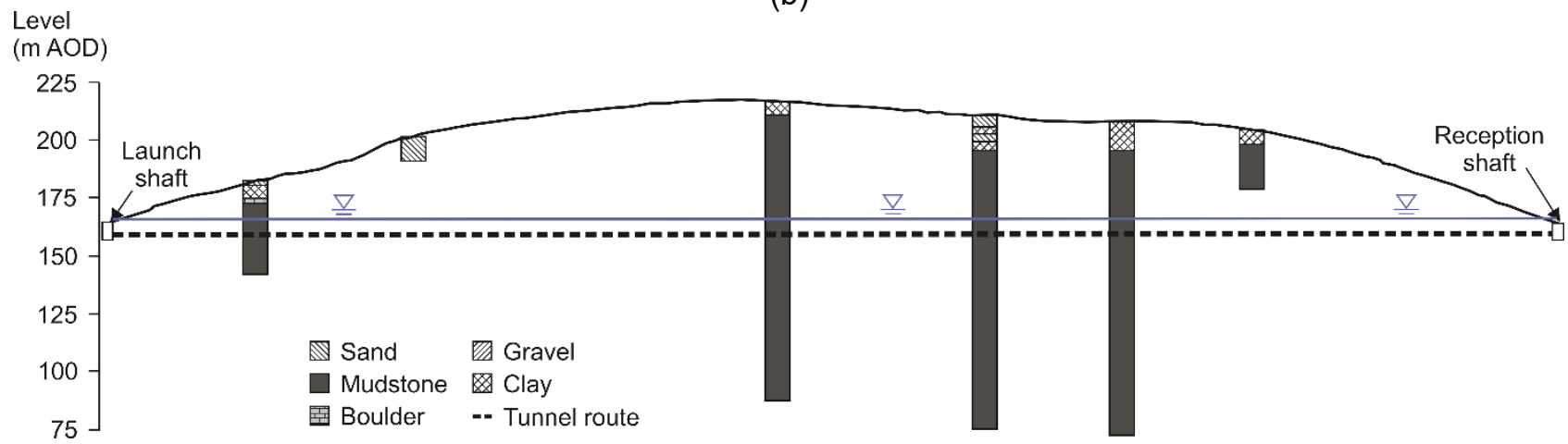

Fig. 9 Case history B: (a) location of tunnels and shafts at the site, (b) borehole profiles along tunnel route

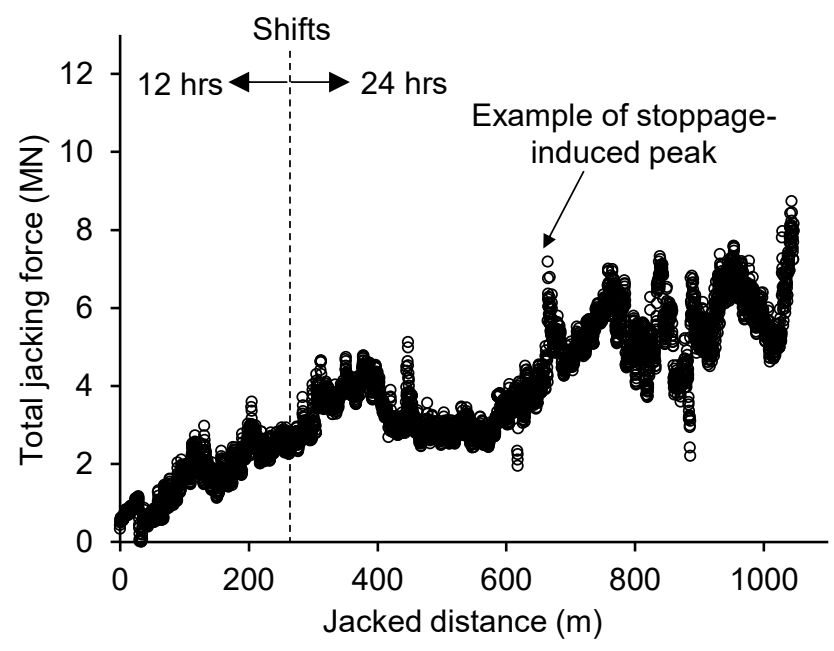

Fig. 10 Monitored data for case history B

(a)

(b) 


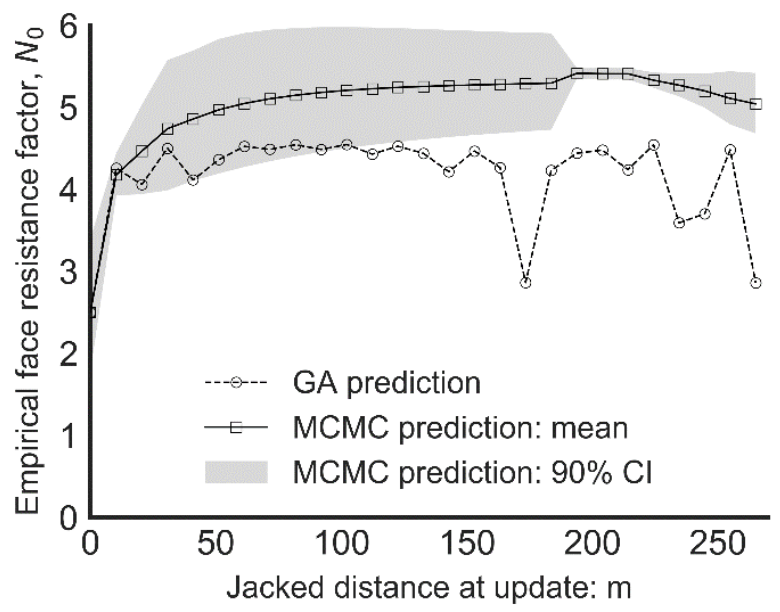

(c)

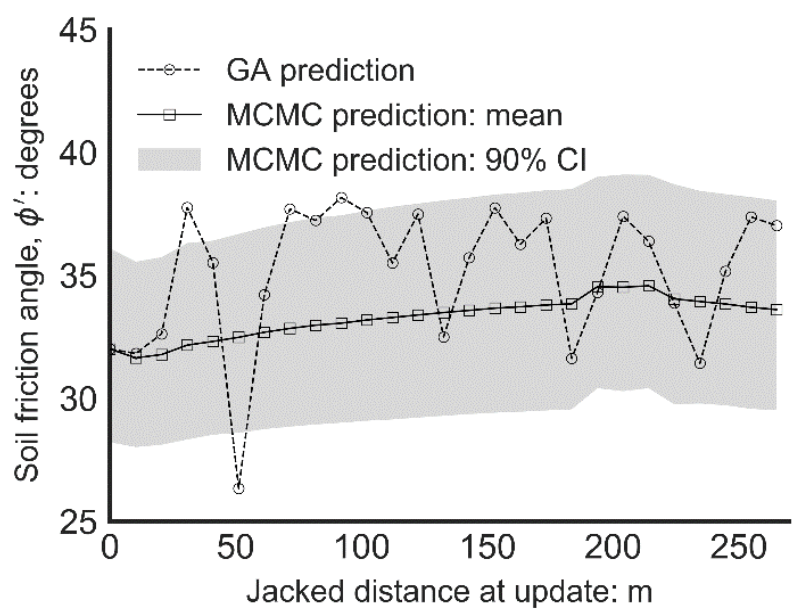

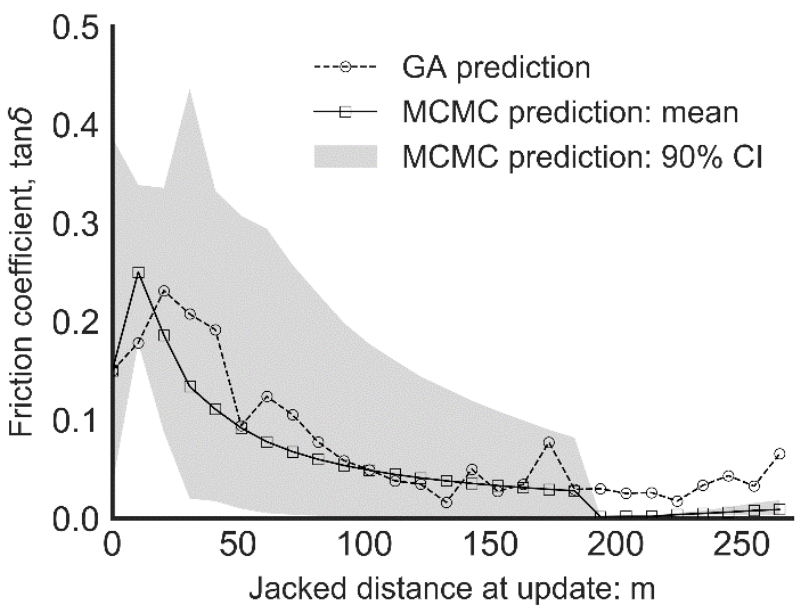

(d)

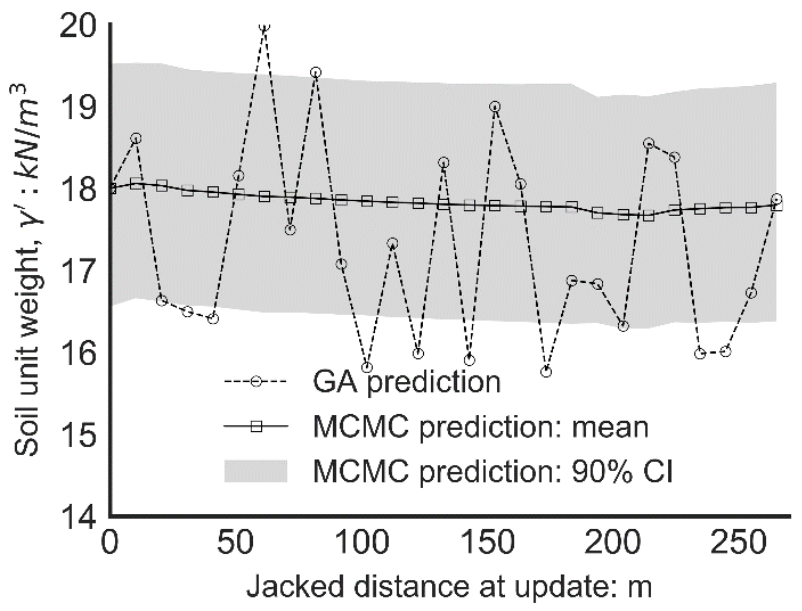

Fig. 11 Comparison of the variation of updated model parameters with jacked distance determined using MCMC and GA approaches for case history A: (a) empirical face resistance factor, $N_{0}$, (b) friction coefficient, tan $\delta,(c)$ friction angle, $\phi^{\prime}$, and (d) soil unit weight, $\gamma^{\prime}$.

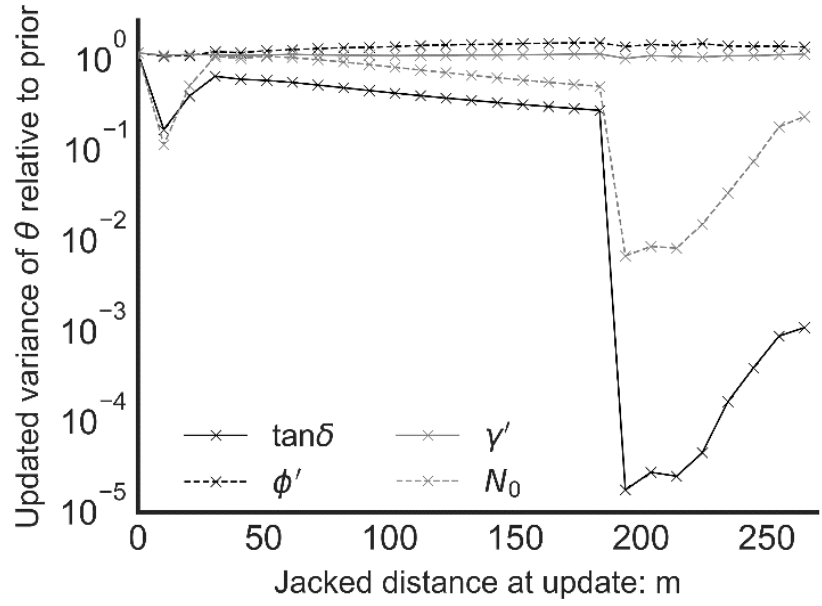

Fig. 12 Updating of the coefficient of variation during the drive for case history A using MCMC for all uncertain parameters

(a)

(b) 


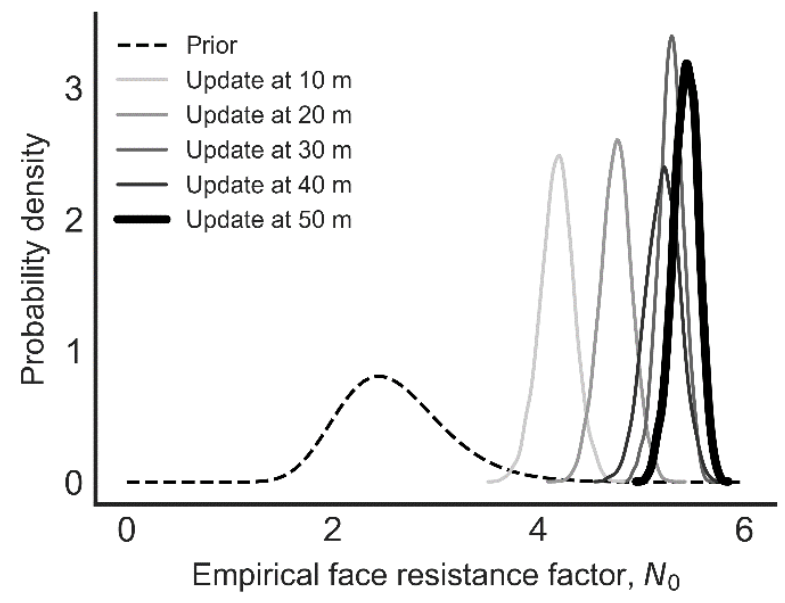

(c)

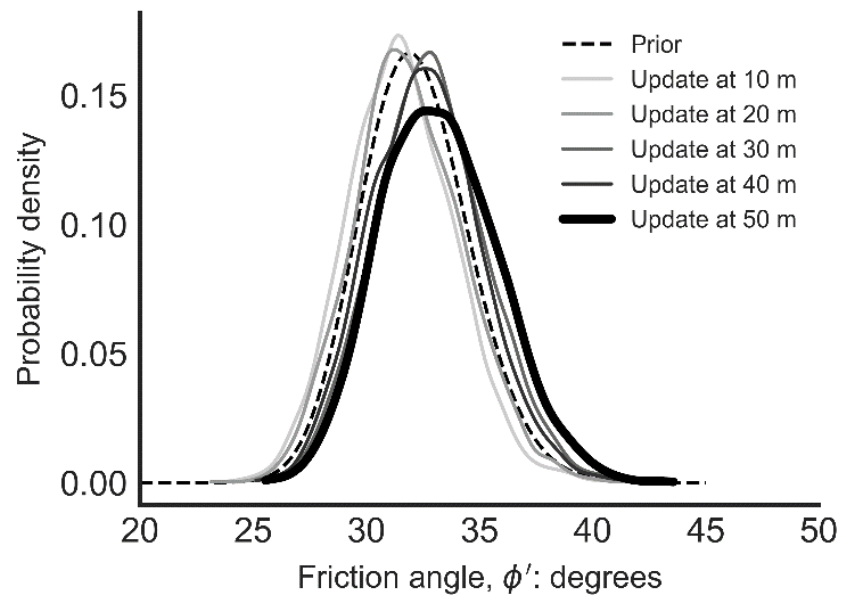



(d)

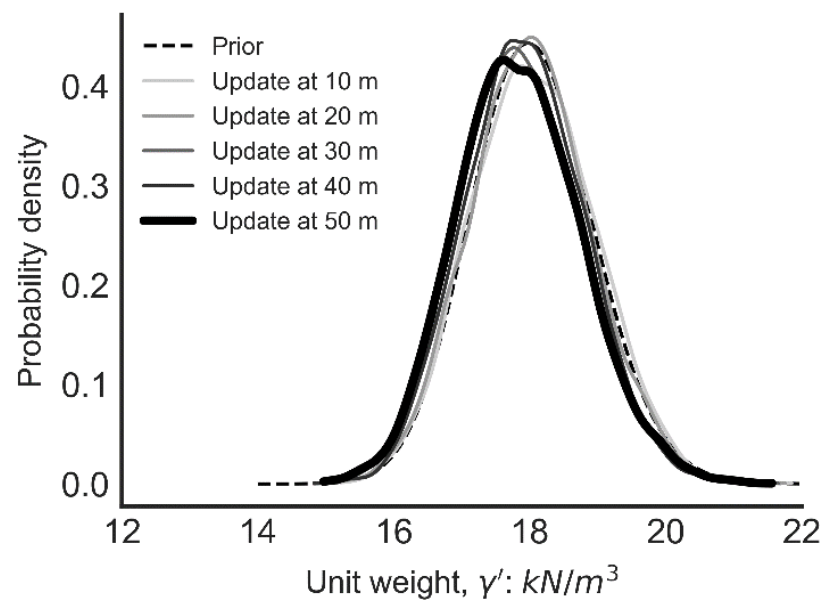

Fig. 13 Result of Bayesian updating process showing updated distributions of uncertain parameters at various stages during pipe jacking for case history $A$ : (a) empirical face resistance factor, $N_{0}$, (b) friction coefficient, tanס, (c) friction angle, $\phi^{\prime}$, and (d) soil unit weight, $\gamma^{\prime}$. 
(a)



(c)

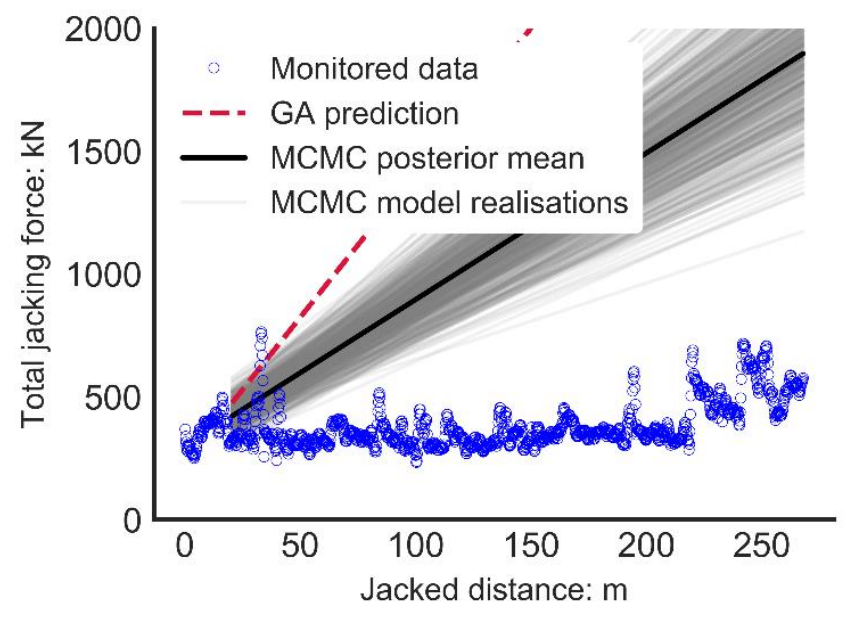

(e)

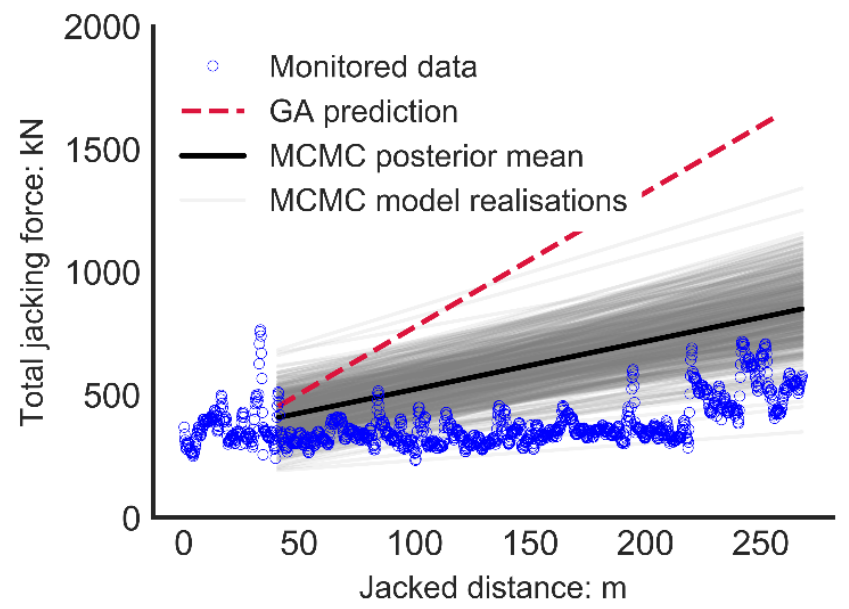

(b)

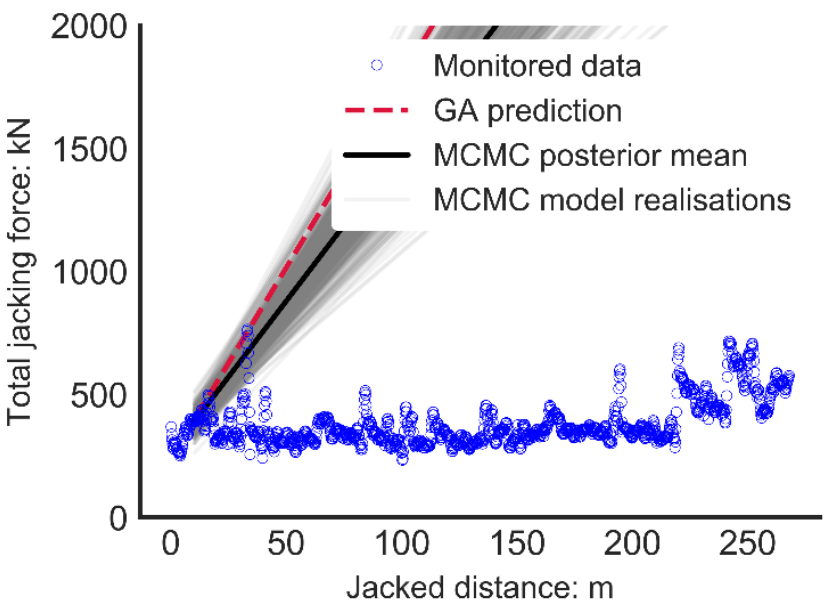

(d)

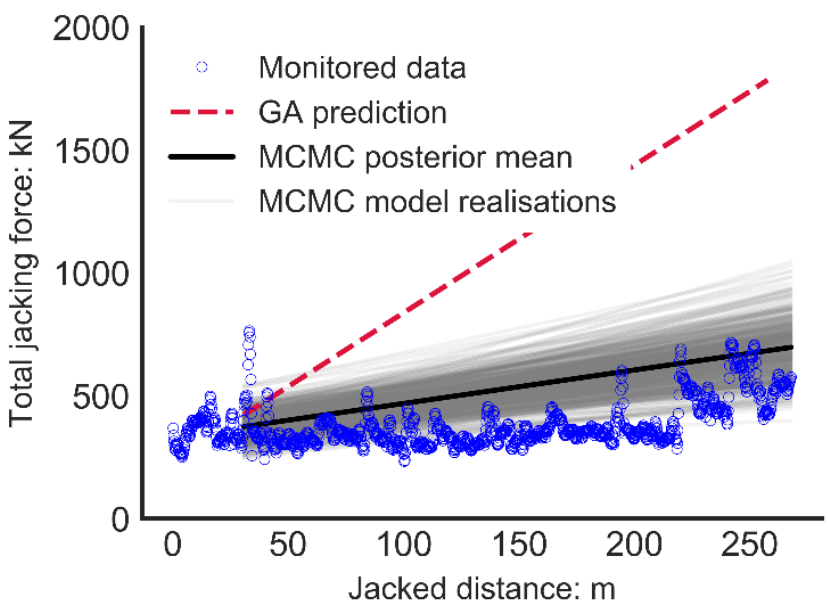

(f)

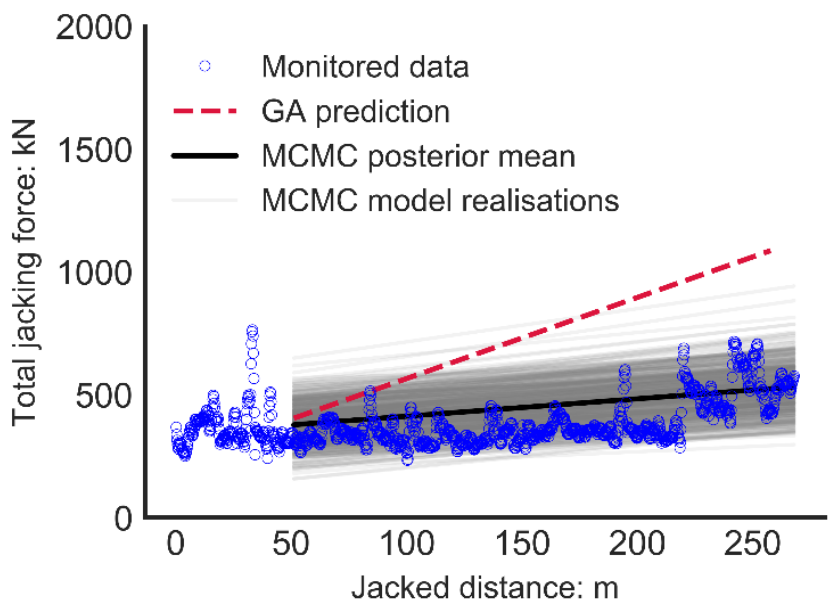

Fig. 14 Model predictions of total pipe jacking forces at various jacked distances for case history A: (a) prior predictions (at $0 \mathrm{~m}$ ), and posterior predictions at (b) $10 \mathrm{~m}$, (c) $20 \mathrm{~m}$, (d) $30 \mathrm{~m}$, (e) $40 \mathrm{~m}$ and (f) $50 \mathrm{~m}$. Model realisations represent predictions determined from 500 draws of the posterior distribution of $\boldsymbol{\theta}$ 
(a)

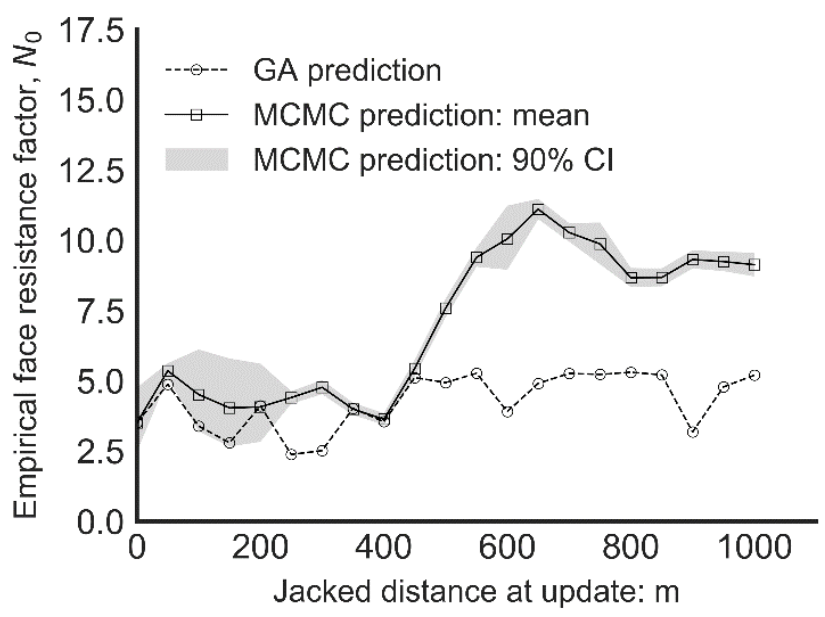

(c)

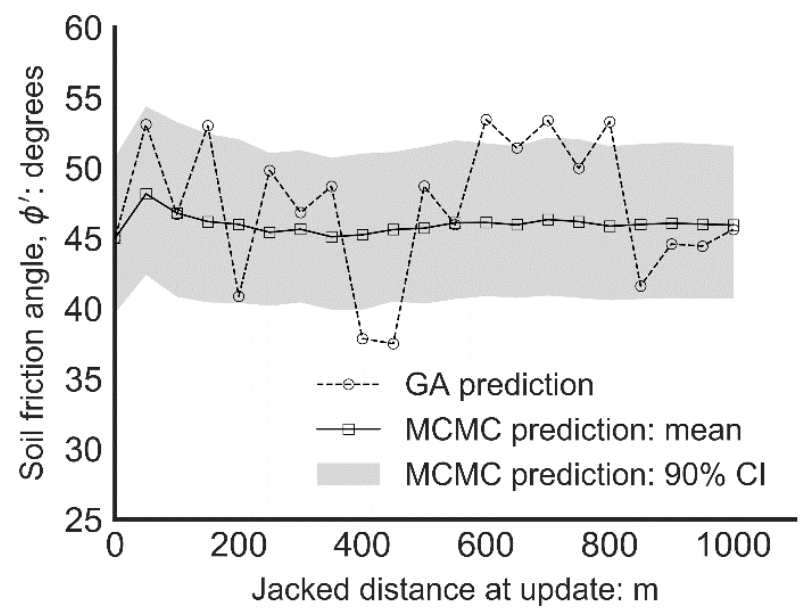

(b)

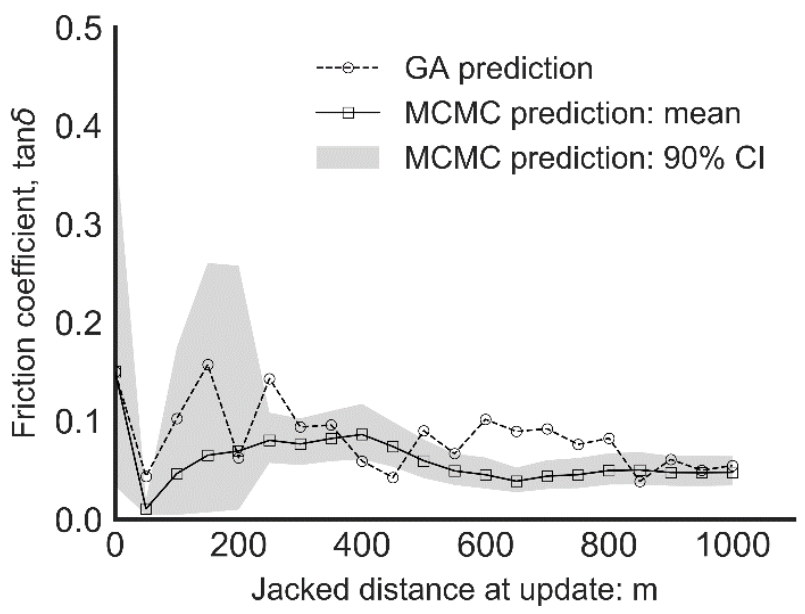

(d)

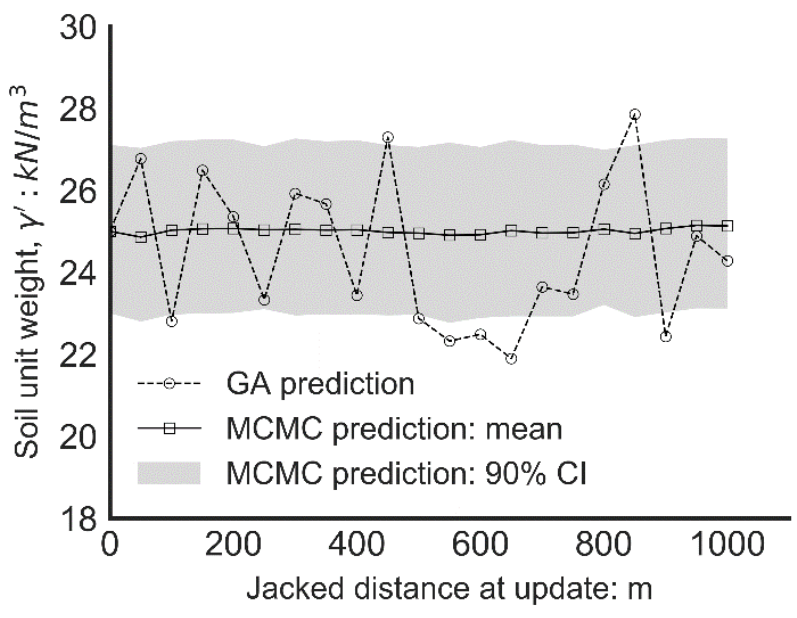

Fig. 15 Comparison of the variation of updated model parameters with jacked distance determined using MCMC and GA approaches for case history B: (a) empirical face resistance facto, $N_{0}$, (b) friction coefficient, tan $\delta,(c)$ friction angle, $\phi^{\prime}$ and (d) soil unit weight, $\gamma^{\prime}$. 


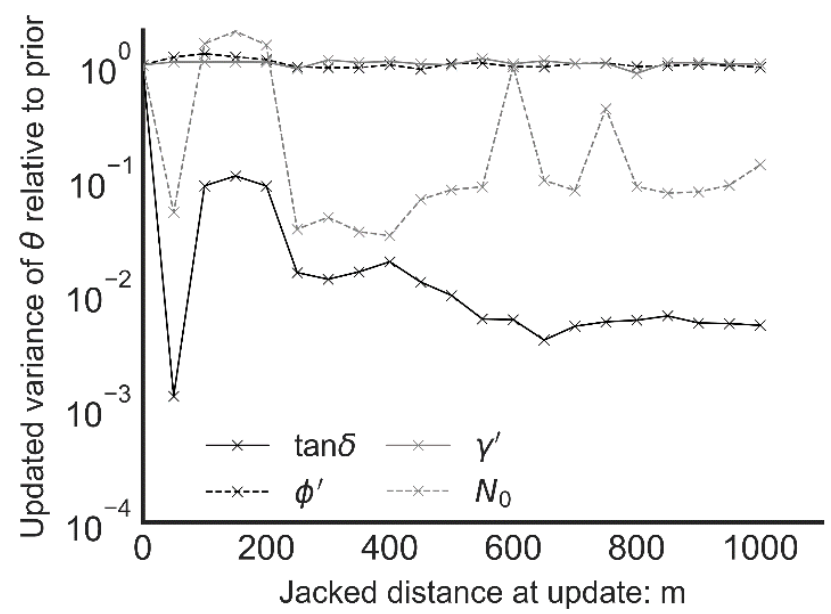

Fig. 16 Updating of the coefficient of variation during the drive for case history B using $\mathrm{MCMC}$ for all uncertain parameters 
(a)

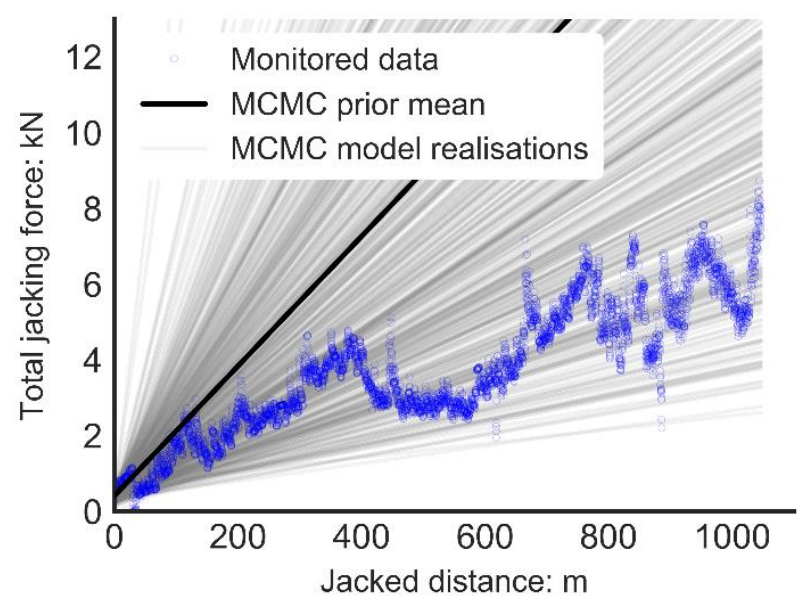

(c)

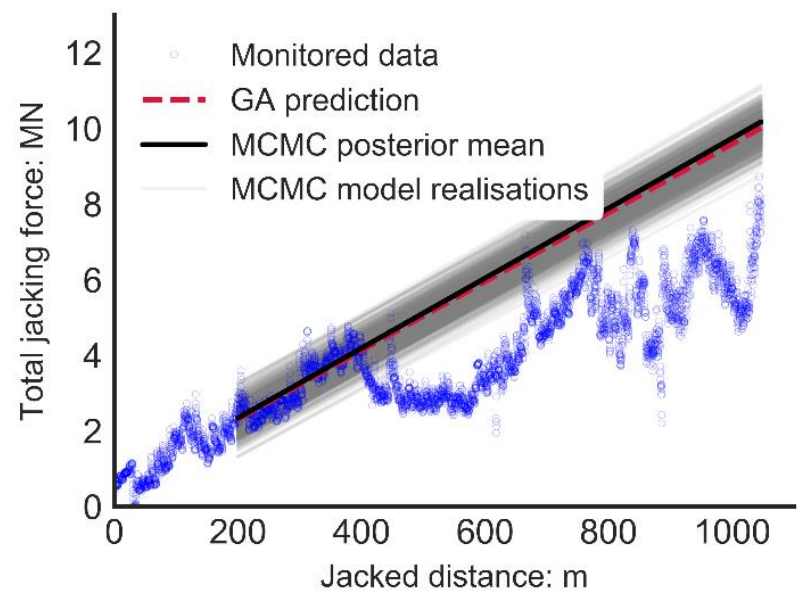

(e)

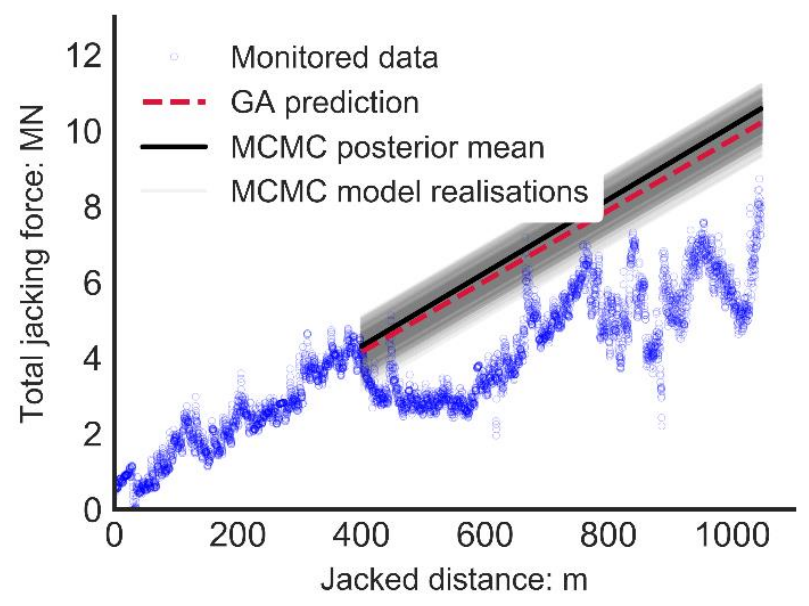

(b)

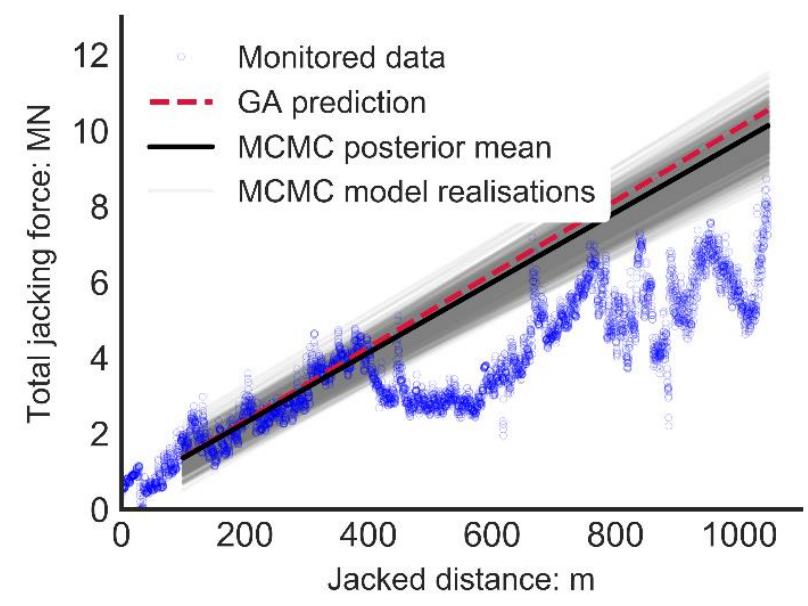

(d)

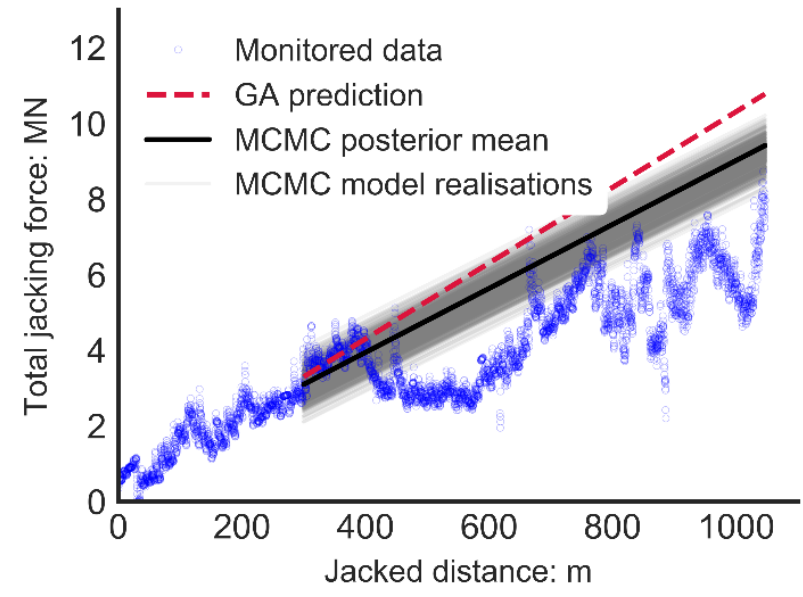

(f)

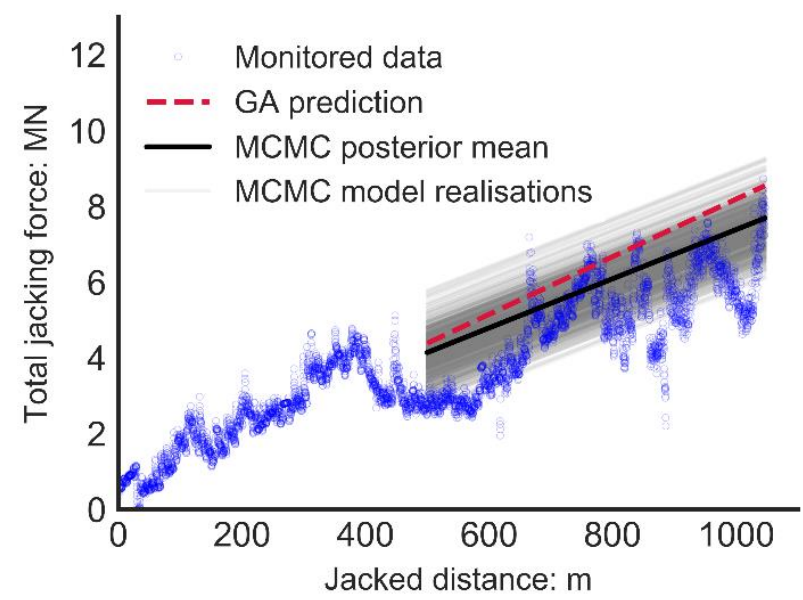

Fig. 17 Model predictions of total pipe jacking forces at various jacked distances for case history B:

(a) prior predictions (at $0 \mathrm{~m}$ ), and posterior predictions at (b) $100 \mathrm{~m}$, (c) $200 \mathrm{~m}$, (d) $300 \mathrm{~m}$, (e) 400 $\mathrm{m}$ and (f) $500 \mathrm{~m}$. Model realisations represent predictions determined from 500 draws of the posterior distribution of $\boldsymbol{\theta}$ 